\title{
Fast track surgery versus conventional recovery strategies for colorectal surgery (Review)
}

\author{
Spanjersberg WR, Reurings J, Keus F, van Laarhoven CJHM
}

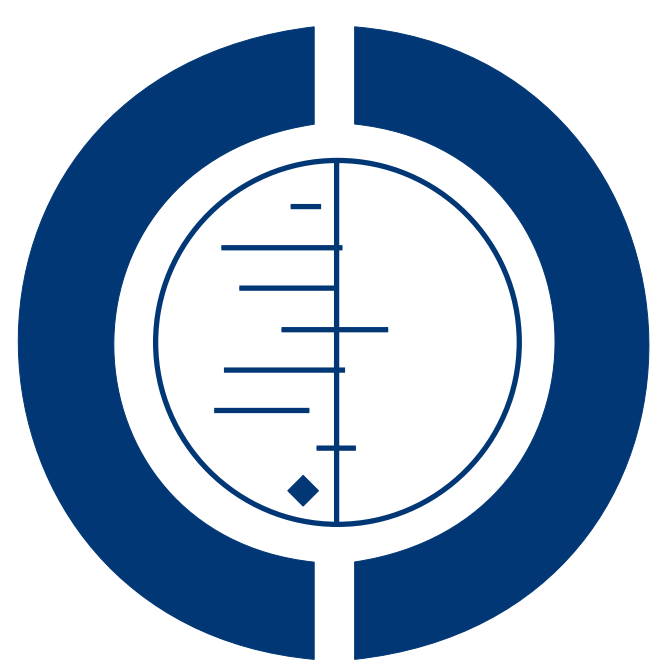

\section{THE COCHRANE COLLABORATION ${ }^{\circledR}$}

This is a reprint of a Cochrane review, prepared and maintained by The Cochrane Collaboration and published in The Cochrane Library 2011, Issue 2

http://www.thecochranelibrary.com

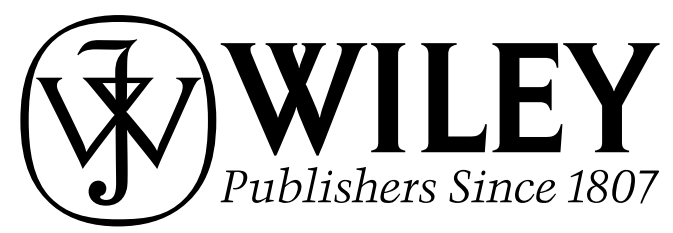

Fast track surgery versus conventional recovery strategies for colorectal surgery (Review)

Copyright $\odot 20$ II The Cochrane Collaboration. Published by John Wiley \& Sons, Ltd. 
TABLE OF CONTENTS

HEADER

PLAIN LANGUAGE SUMMARY . . . . . . . . . . . . . . . . . . . . . . . . . . . . . . . . . . . .

SUMMARY OF FINDINGS FOR THE MAIN COMPARISON . . . . . . . . . . . . . . . . . . . . . . 2

BACKGROUND . . . . . . . . . . . . . . . . . . . . . . . . . . . . . . . . . . . . . . . . . 5

OBJECTIVES . . . . . . . . . . . . . . . . . . . . . . . . . . . . . . . . . . . . . . . . . . . . . . .

METHODS . . . . . . . . . . . . . . . . . . . . . . . . . . . . . . . . . 5

Figure 1. . . . . . . . . . . . . . . . . . . . . . . . . . . . . . . . . . . . 7

RESULTS . . . . . . . . . . . . . . . . . . . . . . . . . . . . . . . . 9

Figure 2. . . . . . . . . . . . . . . . . . . . . . . . . . . . . . . . . . . . . . 10

Figure 3. . . . . . . . . . . . . . . . . . . . . . . . . . . . . . . . . . . . . . 11

Figure $4 . \quad$. . . . . . . . . . . . . . . . . . . . . . . . . . . . . . . . . . . . . 12

Figure 5. . . . . . . . . . . . . . . . . . . . . . . . . . . . . . . . . . . . . . 13

Figure 6. . . . . . . . . . . . . . . . . . . . . . . . . . . . . . . . . . . . . . 13

Figure 7. . . . . . . . . . . . . . . . . . . . . . . . . . . . . . . . . 14

Figure 8. . . . . . . . . . . . . . . . . . . . . . . . . . . . . . . . . . . 14

Figure 9. . . . . . . . . . . . . . . . . . . . . . . . . . . . . . . . . . . 14

Figure 10. . . . . . . . . . . . . . . . . . . . . . . . . . . . . . . . . . . . . . 15

Figure 11. . . . . . . . . . . . . . . . . . . . . . . . . . . . . . . . . . . . . . 16

Figure 12. . . . . . . . . . . . . . . . . . . . . . . . . . . . . . . . . . . . . . 17

Figure 13. . . . . . . . . . . . . . . . . . . . . . . . . . . . . . . . . . . . . . 17

Figure 14. . . . . . . . . . . . . . . . . . . . . . . . . . . . . . . . . . . . . . 17

Figure 15. . . . . . . . . . . . . . . . . . . . . . . . . . . . . . . . . . . . . 18

Figure $16 . \quad$. . . . . . . . . . . . . . . . . . . . . . . . . . . . . . . . . . . . . . . . . . .

DISCUSSION . . . . . . . . . . . . . . . . . . . . . . . . . . . . . . . . . . . . . . . . .

AUTHORS' CONCLUSIONS . . . . . . . . . . . . . . . . . . . . . . . . . . . . . . . . . . . . . . . .

REFERENCES . . . . . . . . . . . . . . . . . . . . . . . . . . . . . . . . . . . . . . 20

CHARACTERISTICS OF STUDIES . . . . . . . . . . . . . . . . . . . . . . . . . . . . . . . . . 23

DATA AND ANALYSES . . . . . . . . . . . . . . . . . . . . . . . . . . . . . . . . . . . . . . . . . . . . . . . . .

Analysis 1.1. Comparison 1 Primary analyses ERAS versus conventional, Outcome 1 Mortality. . . . . . . . . . . 33

Analysis 1.2. Comparison 1 Primary analyses ERAS versus conventional, Outcome 2 All complications. . . . . . 34

Analysis 1.3. Comparison 1 Primary analyses ERAS versus conventional, Outcome 3 Major Complications. . . . 35

Analysis 1.4. Comparison 1 Primary analyses ERAS versus conventional, Outcome 4 Minor complications. . . . . 36

Analysis 1.5. Comparison 1 Primary analyses ERAS versus conventional, Outcome 5 Undefined complications. . . 36

Analysis 1.6. Comparison 1 Primary analyses ERAS versus conventional, Outcome 6 Readmissions. . . . . . . . 37

Analysis 1.7. Comparison 1 Primary analyses ERAS versus conventional, Outcome 7 hospital stay. . . . . . . . . 38

Analysis 2.1. Comparison 2 Sensitivity analyses ERAS vs conventional, Outcome 1 mortality. . . . . . . . . . . . 39

Analysis 2.2. Comparison 2 Sensitivity analyses ERAS vs conventional, Outcome 2 Total complications. . . . . . . 40

Analysis 2.3. Comparison 2 Sensitivity analyses ERAS vs conventional, Outcome 3 Major complications. . . . . . 41

Analysis 2.4. Comparison 2 Sensitivity analyses ERAS vs conventional, Outcome 4 Minor complications. . . . . . 42

Analysis 2.5. Comparison 2 Sensitivity analyses ERAS vs conventional, Outcome 5 Undefined complications. . . . 42

Analysis 2.6. Comparison 2 Sensitivity analyses ERAS vs conventional, Outcome 6 readmissions. . . . . . . . . . 43

Analysis 2.7. Comparison 2 Sensitivity analyses ERAS vs conventional, Outcome 7 hospital stay. . . . . . . . . . 44

ADDITIONAL TABLES . . . . . . . . . . . . . . . . . . . . . . . . . . . . . . . . . . . 44

HISTORY . . . . . . . . . . . . . . . . . . . . . . . . . . . . . . . . . . . . . . . . . 46

CONTRIBUTIONS OF AUTHORS . . . . . . . . . . . . . . . . . . . . . . . . . . . . . . . . . . . . . . . . . . .

DECLARATIONS OF INTEREST . . . . . . . . . . . . . . . . . . . . . . . . . . . . . . . . . . . . . . . . . . 47

DIFFERENCES BETWEEN PROTOCOL AND REVIEW . . . . . . . . . . . . . . . . . . . . . . . . . . . . . . 47

INDEX TERMS . . . . . . . . . . . . . . . . . . . . . . . . . . . . . . . . . . . . . . . . . . . . . . . 47

Fast track surgery versus conventional recovery strategies for colorectal surgery (Review)

Copyright $\odot 2011$ The Cochrane Collaboration. Published by John Wiley \& Sons, Ltd. 


\title{
[Intervention Review] \\ Fast track surgery versus conventional recovery strategies for colorectal surgery
}

\author{
Willem R Spanjersberg ${ }^{1}$, Jurrian Reurings ${ }^{2}$, Frederik Keus ${ }^{1}$, Cornelis JHM van Laarhoven ${ }^{1}$ \\ ${ }^{1}$ Department of Surgery, Radboud University Nijmegen Medical Center, Nijmegen, Netherlands. ${ }^{2}$ Department of Surgery, St. Elisabeth \\ Ziekenhuis, Tilburg, Netherlands
}

Contact address: Willem R Spanjersberg, Department of Surgery, Radboud University Nijmegen Medical Center, PO Box 9101, Nijmegen, 6500 HB, Netherlands. w.r.spanjersberg@gmail.com.

Editorial group: Cochrane Colorectal Cancer Group.

Publication status and date: New, published in Issue 2, 2011.

Review content assessed as up-to-date: 3 January 2011.

Citation: Spanjersberg WR, Reurings J, Keus F, van Laarhoven CJHM. Fast track surgery versus conventional recovery strategies for colorectal surgery. Cochrane Database of Systematic Reviews 2011, Issue 2. Art. No.: CD007635. DOI: 10.1002/14651858.CD007635.pub2.

Copyright (C) 2011 The Cochrane Collaboration. Published by John Wiley \& Sons, Ltd.

\begin{abstract}
A B S T R A C T
Background

In recent years the Enhanced Recovery after Surgery (ERAS) postoperative pathway in (ileo-)colorectal surgery, aiming at improving perioperative care and decreasing postoperative complications, has become more common.
\end{abstract}

\section{Objectives}

We investigated the effectiveness and safety of the ERAS multimodal strategy, compared to conventional care after (ileo-)colorectal surgery. The primary research question was whether ERAS protocols lead to less morbidity and secondary whether length of stay was reduced.

\section{Search methods}

To answer the research question we entered search strings containing keywords like "fast track", "colorectal and surgery" and "enhanced recovery" into major databases. We also hand searched references in identified reviews concerning ERAS.

\section{Selection criteria}

We included published randomised clinical trials, in any language, comparing ERAS to conventional treatment in patients with (ileo) colorectal disease requiring a resection. RCT's including at least 7 ERAS items in the ERAS group and no more than 2 in the conventional arm were included.

\section{Data collection and analysis}

Data of included trials were independently extracted by the reviewers. Analyses were performed using "REVMAN 5.0.22". Data were pooled and rate differences as well as weighted mean differences with their $95 \%$ confidence intervals were calculated using either fixed or random effects models, depending on heterogeneity $\left(\mathrm{I}^{2}\right)$.

Fast track surgery versus conventional recovery strategies for colorectal surgery (Review)

Copyright (c) 201 I The Cochrane Collaboration. Published by John Wiley \& Sons, Ltd. 


\section{Main results}

4 RCTs were included and analysed. Methodological quality of included studies was considered low, when scored according to GRADE methodology. Total numbers of inclusion were limited. The trials included in primary analysis reported 237 patients, (119 ERAS vs 118 conventional). Baseline characteristics were comparable. The primary outcome measure, complications, showed a significant risk reduction for all complications (RR $0.50 ; 95 \%$ CI 0.35 to 0.72 ). This difference was not due to reduction in major complications. Length of hospital stay was significantly reduced in the ERAS group (MD -2.94 days; 95\% CI -3.69 to -2.19), and readmission rates were equal in both groups. Other outcome parameters were unsuitable for meta-analysis, but seemed to favour ERAS.

\section{Authors' conclusions}

The quantity and especially quality of data are low. Analysis shows a reduction in overall complications, but major complications were not reduced. Length of stay was reduced significantly. We state that ERAS seems safe, but the quality of trials and lack of sufficient other outcome parameters do not justify implementation of ERAS as the standard of care. Within ERAS protocols included, no answer regarding the role for minimally invasive surgery (i.e. laparoscopy) was found. Furthermore, protocol compliance within ERAS programs has not been investigated, while this seems a known problem in the field. Therefore, more specific and large RCT's are needed.

\section{PLAIN LANGUAGESUMMARY}

\section{Fast track surgery versus conventional recovery strategies for colorectal surgery}

Conventionally, recuperation after bowel surgery followed the patients progress. Mobilisation and expansion of diet after surgery was progressed slowly in a stepwise manner following patients progression. This is because it was believed that faster recovery would be unwise. In recent years, however, a new concept has been introduced, called Enhanced Recovery after surgery (ERAS) or fast track. This program, introduced by Kehlet et al, is based on the principle that reducing the body's stress response after surgery reduces the time needed to recuperate. This is achieved by interventions around the operation, involving good information, better feeding before the operation and better pain treatment, so patients can get out of bed earlier and start a normal diet earlier and thereby reducing the risk of complications. This review investigated whether this intervention is safe and whether it is more effective than the traditional treatment. In order to answer this question, 4 randomised trials were found, comparing these two interventions. We found that ERAS can be viewed as safe, i.e. not resulting in more complications or deaths, and at the same time decreases the days spent in hospital following major bowel surgery. However, the data are of low quality and therefore does not justify implementation of ERAS as the standard method of care yet. More research on other outcome parameters like economical evaluation and quality of life parameters are necessary. 


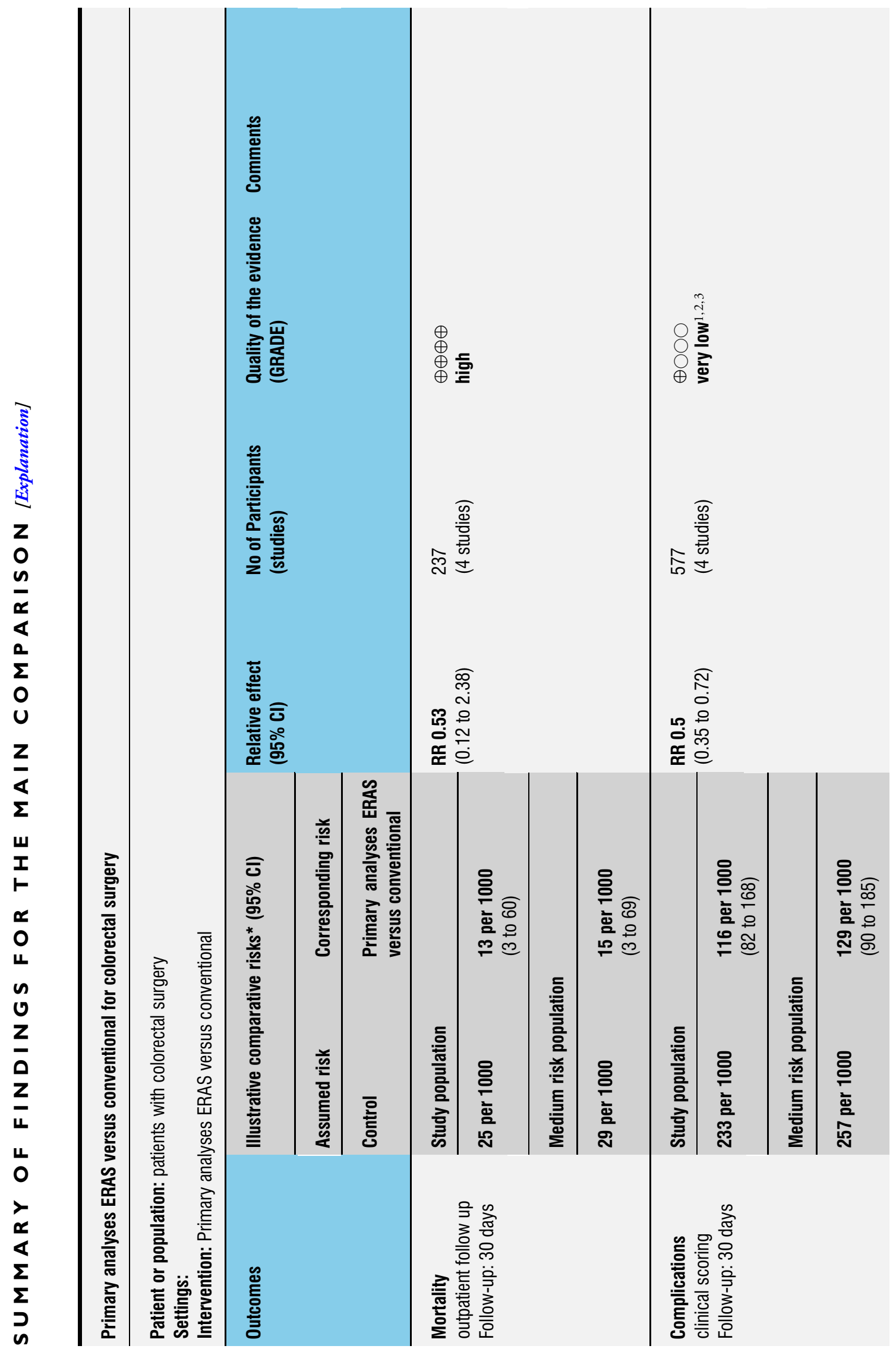

Fast track surgery versus conventional recovery strategies for colorectal surgery (Review)

Copyright $\odot 201$ I The Cochrane Collaboration. Published by John Wiley \& Sons, Ltd. 


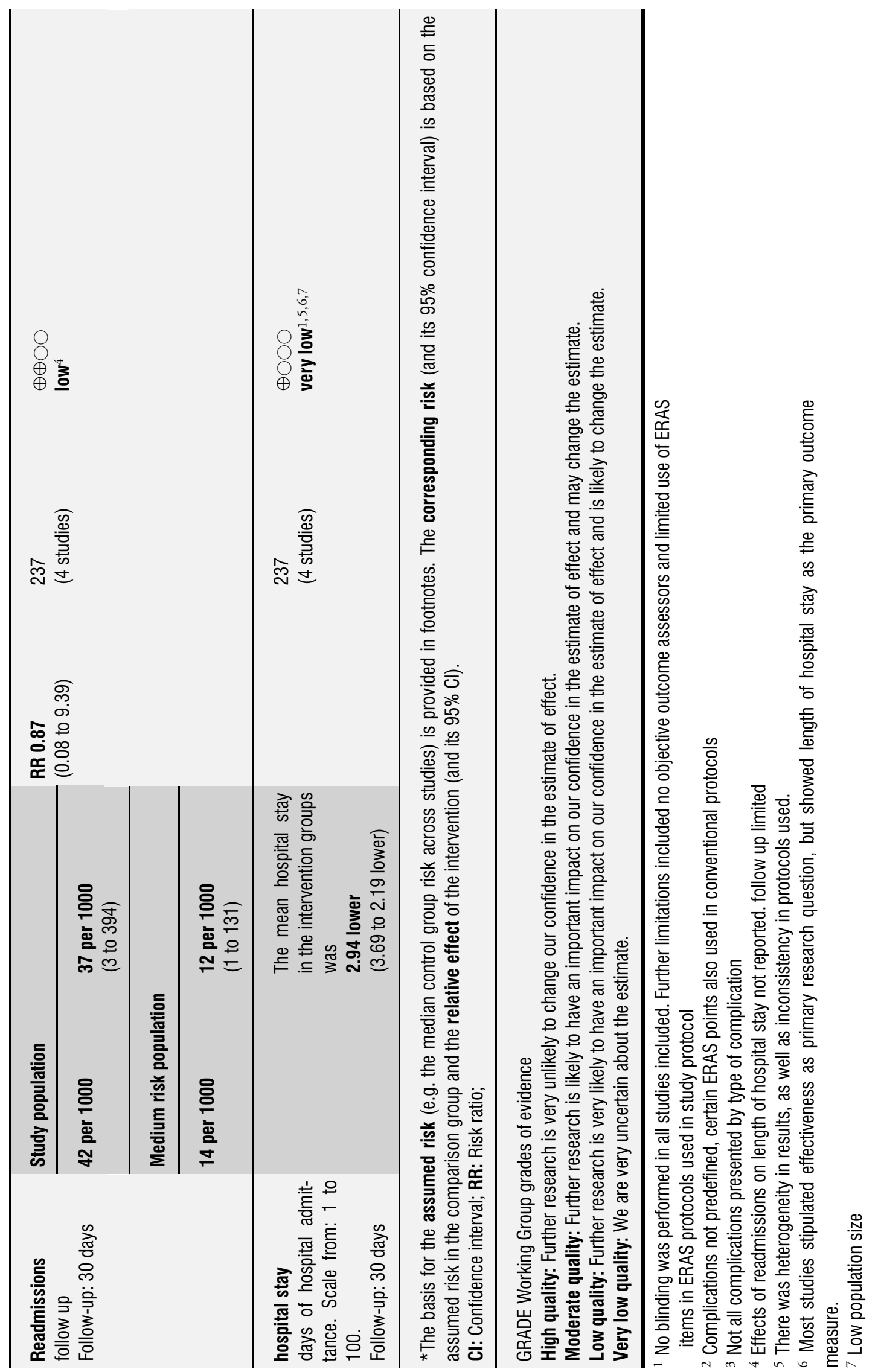

Fast track surgery versus conventional recovery strategies for colorectal surgery (Review)

Copyright $\odot 201$ I The Cochrane Collaboration. Published by John Wiley \& Sons, Ltd. 


\section{B A C K G RO U N D}

\section{Description of the condition}

Colorectal carcinoma is one of the malignancies with the highest incidence in the world and surgery is the main treatment modality (Weitz 2005). Besides malignancy, other benign conditions such as diverticulitis and inflammatory bowel disease often require surgery. Therefore major abdominal surgery with small bowel or colorectal resections have a high incidence. Surgical approach may be the conventional open procedure or an laparoscopic resection. Complication rates after resections, either open or laparoscopic, are reported as high as $15 \%$ and $20 \%$ respectively (Tjandra 2006). Traditionally, the length of hospital stay in recent large trials varies between 5 days for laparoscopic surgery and 6 days for open surgery in the COST trial (Weeks 2002) and $8.2(+/-6.6)$ days for laparoscopic surgery and $9.3(+/-7.3)$ days for open surgery in the COLOR trial (Hazebroek 2002). Main reasons for increasing length of clinical postoperative treatment are pain, nausea and persistent ileus (Basse 2000; Basse 2002; Anderson 2003; Kehlet 2003; Basse 2004).

\section{Description of the intervention}

In recent years, a trend towards new peri-operative treatment strategies has been seen; "Fast track surgery" or Enhanced Recovery After Surgery (ERAS). ERAS programs focus on a number of techniques that facilitate early recovery after major surgery by preserving pre-operative bodily composition and organ functions. Techniques include optimal pain control by epidural and local anaesthesia, minimally invasive techniques, and aggressive postoperative rehabilitation (Lassen 2009; Wilmore 2001). All these interventions are chosen on the basis of high-grade evidence of clinical efficacy. The first to incorporate these strategies in elective colonic surgery were Kehlet and associates in the mid 90's, showing a reduction of days to recovery to as early as 2 days postoperatively (Kehlet 2007).

\section{How the intervention might work}

By reducing stress and pain in colorectal resections, together with aggressive postoperative mobilisation and early oral feeding, the body's stress response is reduced and organ dysfunction is limited to a minimum, thus facilitating early recovery and reducing postoperative morbidity and mortality.

\section{Why it is important to do this review}

The implementation of ERAS programs in colorectal surgery is supported by review of controlled trials (Wind 2006) and randomised controlled trials (Khoo 2007). However, the effects of changing ERAS protocols used and the amount of interventions used in these protocols set against the recommended set of 17 interventions are not taken into account (Lassen 2009). For this reason, known RCT's involving ERAS interventions may hold a high risk of bias that may not have been sufficiently appreciated in known meta-analyses. Therefore, by focusing more on quality of found trials and implementing more stringent inclusion criteria we tried to increase the level of evidence concerning ERAS programs in colorectal surgery. With this evidence, the field may be able to make a more evidence based decision on implementation of ERAS protocols. This may well lead to increased or decreased implementation around the world with major implications, both for patients, organisation of health care and economical cost (Kehlet 2008).

\section{O B J E C T I VES}

To evaluate beneficial and harmful effects of ERAS recovery after surgery for colorectal carcinomas and benign conditions, by investigating whether ERAS recovery after colorectal resections differs in primary (complications, both major, minor and mortality, and length of hospital stay, including readmissions) and secondary outcome measures (quality of life, need for re-operation, better physiological function) in reference to conventional recovery. Other outcome measures, such as cost-effectiveness, time to return to work, postoperative need for analgesia etcetera were also investigated.

\section{METHODS}

\section{Criteria for considering studies for this review}

\section{Types of studies}

All randomised clinical trials comparing any type of ERAS recovery strategy for resections in colorectal disease to conventional recovery strategies.

Trials will be included irrespectively of blinding, number of patients randomised, and language of the article. Articles must be published in peer reviewed indexed journals. Because of expected flaws in design and the added risk of bias, only randomised trials were incorporated in this review.

\section{Types of participants}

Patients undergoing resection of any portion of the small bowel, colon or rectum via either laparotomy or laparoscopy. 


\section{Types of interventions}

In this review we will compare any type of "ERAS" recovery strategy with conventional recuperation. ERAS recovery strategies include programs using epidural or local anaesthesia, minimally invasive techniques, optimal pain control and aggressive postoperative rehabilitation to achieve early recovery after colorectal surgery. In total, 17 items are scored, according to the consensus review of the ERAS working group (Lassen 2009). An important problem also to be investigated is the quality of ERAS protocols used in studies, because detailed review of literature suggests that not all studies review actual ERAS protocols, but rather conventional care that has been protocolised. We therefore first scored each protocol using the working groups recommendations and recorded the numbers of items used in each subgroup. We set the debatable limit at least 7 items used in ERAS groups and no more than 2 items in the conventional groups.

\section{Types of outcome measures}

\section{Primary outcomes}

Primary outcomes in choosing between interventions should primarily be medical reasons, i.e. safe(r) and better treatment of disease. The primary outcome parameters in this review therefore will be:

Mortality (both early and late), with early mortality defined as death within 30 days was analysed.

Overall complications, and because of different impacts, these were further divided into both major (including abdominal sepsis, anastomotic leakage, need for reoperation, persistent ileus, intraabdominal abscesses, bleeding, burst abdomen (Platzbauch), late incisional hernia and adhesions) and minor (pneumonia, wound infection, deep vein thrombosis, and urinary tract infection) because safety of this intervention is a major decisional factor in its implementation. No definition for (specific) complications exist, so we acknowledge that definitions between studies may vary. However, for this review we accepted the authors own definitions at face value.

Additionally, although we do not appreciate it as a medically important outcome parameter, but since it is an economical parameter that may prove important, length of hospital stay; both primary and total length of stay (including readmissions) are investigated. This supposition is further supported by GRADE methodology, as presented by Guyatt (Guyatt 2008), in which the importance of an outcome to patients is put as the central perspective to establish importance of outcome parameters. Especially readmissions are an often feared factor in ERAS programs, because certain complications do not present until ERAS patients have already been released from clinical care.

\section{Secondary outcomes}

Secondary outcome measures are all other outcomes assessed in comparing the conventional postoperative protocol with ERAS protocols.

These include operative time, economical evaluation and quality of life. Depending on availability other outcome data like pain scores, analgesic use, and other physiological data are analysed.

\section{Search methods for identification of studies}

The devised search string was entered in the following databases: -The Cochrane Database of Systematic Reviews,

-Database of Abstracts of Reviews of Effects (DARE),

-The Cochrane Central Register of Controlled Trials (CENTRAL),

-NHS Economic Evaluation Database, all in The Cochrane Library (Issue 3, 2008),

-MEDLINE (1985 until present),

-EMBASE (1985 until present) and

-ISI Web of Knowledge (Web of Science) (1985 until present)

-Webcasts of the annual meetings of the American Society of Colon and Rectal Surgeons (ASCRS)

Our aim was to perform a maximal sensitive search in order to perform a more complete review. Our search strategy has been developed in accordance to the guidelines outlined in the Cochrane Handbook for Systematic Reviews of Interventions, chapter 5.2. The last part of this strategy, concerning the sensitive search for randomised and controlled trials, corresponds to the Cochrane Highly Sensitive Search Strategy for Identifying Reports of Randomised Controlled Trials phases 1, $2 \& 3$. We started the search from the year 1985 given that 'ERAS' approaches were not described before 1989 and therefore it would be very unlikely that any relevant trials will be found prior to this year.

\section{Electronic searches}

The specific search strategies that are formed are adapted to the syntax and capacities of each database. The used implementations of our search strategy for the different databases are shown in Table 1.

\section{Searching other resources}

Additional relevant trials by cross-reference checking will be looked for in the reference lists of identified randomised trials. Finally, authors of identified unpublished, or ongoing trials in CENTRAL were contacted to provide relevant preliminary data, but no data were given. Also, all identified (systematic) reviews concerning ERAS in colorectal surgery were retrieved and references were hand searched. 


\section{Data collection and analysis}

The review was conducted according to the prespecified protocol (Spanjersberg 2009) and the recommendations by the Cochrane Handbook for Systematic Reviews of Interventions (Higgins 2008).

\section{Selection of studies}

The titles, abstracts and descriptor terms of all downloaded material from the electronic searches were read by WRS and irrelevant reports discarded. All citations identified were then inspected independently by WRS and by JCR to establish relevance of the article according to the pre-specified criteria. If any uncertainty arose about the relevance of the study, the full article was obtained. Studies were reviewed for relevance based on study design, types of participants, types of interventions and outcome measures.

After identifying relevant articles, WRS and JCR independently applied the inclusion criteria. Differences were resolved by discussion with the third reviewer, CL, and consensus amongst all reviewers was reached. All identified trials are listed in the characteristics of included studies table and excluded trials and the reasons for exclusion are listed as well (characteristics of excluded studies).

\section{Data extraction and management}

Two reviewers (WRS and JR) independently extracted all relevant data using a specifically designed data extraction form. For each study patient characteristics, study characteristics, data needed for the methodological quality assessment of the study and the primary and secondary outcomes were extracted according to availability. Data regarding patient characteristics included number of patients in each group, age, gender, BMI and diagnoses of included patients. Data regarding study characteristics included study design, sample size information, inclusion and exclusion criteria of the study, follow-up period, loss to follow-up, surgical experience and information regarding surgical techniques. For each study data regarding the perioperative interventions in both the Enhanced Recovery ERAS group and conventional group were also extracted. According to the recommendations of the European ERAS Study Group, the ERAS program needs to involve 17 interventions (Lassen 2009). The number of interventions used in both groups for each study was recorded and presented in the Characteristics of included studies. The exact interventions used are graphically depicted in Figure 1. The difference between the number of intervention used between the conventional and ERAS groups has to be large enough in order to judge the effect of the intervention named ERAS. We therefore regarded ERAS protocols implementing 7 or more ERAS items and conventional protocols implementing no more than 2 items to be adequate for comparison.

Figure I. Number of ERAS items used in included studies (intervention group)

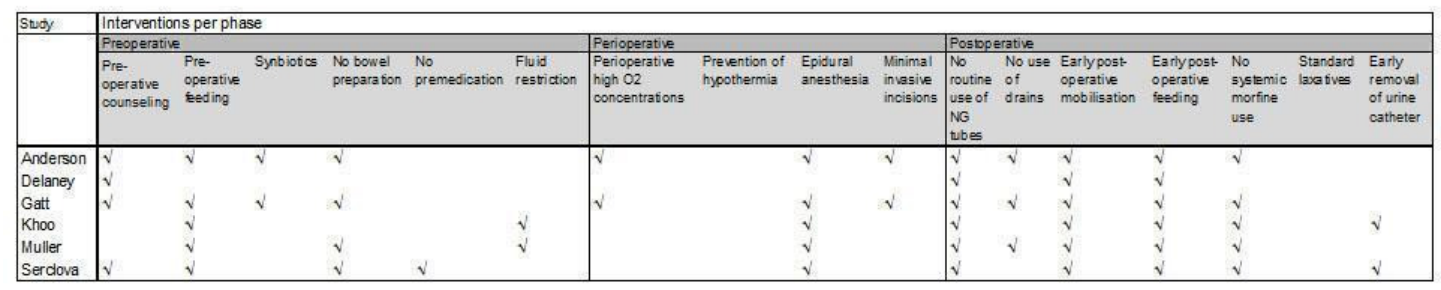

In RCT's the general descriptive data (like gender, age, body mass index (BMI), and American Society of Anaesthesiology (ASA) classification) are supposed to be equally divided due to randomisation. Therefore statistical analysis of patient characteristics in RCT's is not appropriate (Assmann 2000). We did, however, present general descriptive data of included patients in Table 2.

If during data extraction it turns out that essential data or information on methods were missing from certain trials/studies, the authors of those trials or studies were contacted and asked to provide for the missing data. Extracted data was stored and managed using the review manager software package RevMan, version 5.0.23, provided by The Cochrane Collaboration.

\section{Assessment of risk of bias in included studies}

\author{
Assessment of methodological quality of randomised clinical \\ trials \\ Based on the available empirical evidence (Schultz 1995; Moher \\ 1998; Kjaergard 2001; Higgins 2008) the methodological quality \\ of RCTs was assessed using the following items and incorporated \\ in the Characteristics of included studies section. \\ Generation of the allocation sequence \\ Adequate, if the allocation sequence was generated by a computer
}


or random number table. Drawing of lots, tossing of a coin, shuffling of cards, or throwing dice was considered as adequate if a person who was not otherwise involved in the recruitment of participants performed the procedure.

Unclear, if the trial was described as randomised, but the method used for generation of the allocation sequence was not described or the sequence had an higher risk of bias (like simply randomising by opening envelopes).

Inadequate, if a system involving dates, names, or admittance numbers was used for the allocation of patients.

Allocation concealment

Adequate, if the allocation of patients involved a central independent unit, on-site locked computer, or sealed envelopes opened by independent assessors.

Unclear, if the trial was described as randomised, but the method used to conceal the allocation was not described.

Inadequate, if the allocation sequence was known to the investigators who assigned participants or if the study was quasi-randomised.

Blinding

Adequate, if the trial was described (at least) as blind to participants or assessors and the method of blinding was described. We are well aware that it is very difficult to properly blind trials comparing surgical treatments, therefore one level of blinding was considered adequate.

Unclear, if the trial was described as (double) blind, but the method of blinding was not described.

Not performed, if the trial was not blinded.

Follow-up

Adequate, if the numbers and reasons for dropouts and withdrawals in all intervention groups were described or if it was specified that there were no dropouts or withdrawals.

Unclear, if the report gave the impression that there had been no dropouts or withdrawals, but this was not specifically stated.

Inadequate, if the number or reasons for dropouts and withdrawals were not described.

These items were scored to assess bias and are depicted as methodological summary graph and summary. Further analysis on the risk of bias and thereby quality of the evidence was performed using the Grade profiler tool, as provided by the Cochrane collaboration. (GRADEprofiler, v3.2.2, (C) Grade working group, 20042007). Results are shown in Summary of findings for the main comparison In order to assess the risk of publication bias, funnel plots were created (Figure 3), and scoring amended accordingly.

\section{Measures of treatment effect}

With adequate data available statistical analysis of binary data will be conducted using relative risks (RR) as the summary statistic. Trials with zero events in both arms are to be excluded from metaanalyses. However, a sensitivity analysis using risk differences (RD) can be performed with inclusion of these trials, and in case of inconsistency the results of this sensitivity analysis reported (Keus 2009).

For continuous outcomes weighted mean differences (WMD) were used as the summary statistic. Authors, however, often presented their results in medians with ranges due to suspicion of skewed data, while means with their standard deviations (SD) are needed for meta-analysis. Then, sensitivity analyses imputing data for missing means and standard deviations (calculated from available medians and ranges) was performed (Hozo 2005).

\section{Dealing with missing data}

In analysis of data, missing of data is of importance. In case of missing data we investigated whether this data was missing at random, in which case the missing data was not regarded as being of influence on outcome, or data missing not at random, in which case missing data had to be obtained. Finally, no additional data had to be obtained.

\section{Assessment of heterogeneity}

The main focus of looking at heterogeneity in meta-analysis is to discriminate true effect modifiers from other sources of heterogeneity. Heterogeneity is calculated by the Cochrane Q test and quantified by measuring I2. If excessive heterogeneity is detected, data will be re-checked first and then adjusted. Extreme outliers will be excluded (and tested in sensitivity analyses) when adequate reasons are available. If excessive heterogeneity still remains, depending on the specific research question, alternative methods will be considered: subgroup analysis and meta-regression if appropriate. Heterogeneity was calculated using Higgins chi-square test and quantified by measuring $I^{2}$ (Higgins 2002). A chi-square test with a P-value of $<0.10$ was considered to indicate the presence of heterogeneity, while an $\mathrm{I}^{2}>50 \%$ was considered to suggest a marked inconsistency in effect between studies. In case of no discrepancy (and no heterogeneity) the fixed-effect models is presented. The fixed-effect model was only used if no or low heterogeneity was present $\left(\mathrm{I}^{2}<25 \%\right)$. In all other cases the random-effects model was used. Both the random-effects model (Dersimonian 1986) and the fixed-effect model (Demets 1987) for pooling effect estimates were explored.

In case of discrepancy between the two models (e.g., one giving a significant intervention effect and the other no significant intervention effect) both results are reported. Discrepancy will only occur when substantial heterogeneity is present.

Most weight will be put on the results of the fixed-effect model if the meta-analysis includes one or more large trials, provided that they have adequate methodology. (By large trials we mean trials that outnumber the rest of the included trials in terms of numbers of outcomes and participants (e.g., more than half of all included events and participants)). 
Otherwise, most weight will be put on the results of the randomeffects model as it incorporates heterogeneity. The reason for this is that the random-effects model increases the weight of small trials. Small trials however are more often than large trials conducted with unclear or inadequate methods (Kjaergard 2001).

Finally, in situations of excessive heterogeneity we refrained from reporting a pooled estimate when inappropriate.

\section{Assessment of reporting biases}

We used funnel plots to provide a visual assessment of whether treatment estimates are associated with study size. The presence of publication bias and other biases (Begg 1994; Egger 1997; Macaskill 2001) varies with the magnitude of the treatment effect, the distribution of study size, and whether a one- or two-tailed test is used (Macaskill 2001).

\section{Subgroup analysis and investigation of heterogeneity}

If excessive heterogeneity was present, data were re-checked first. If heterogeneity persisted, subgroup or sensitivity analyses were used to explore its causes. When adequate reasons were present extreme outliers were excluded in sensitivity analyses. In situations of excessive heterogeneity that could not be explained, we refrained from reporting a pooled estimate.

Subgroup analyses was performed to compare the effects of the interventions according to the methodological quality of the trials, i.e. low level RCT's were included in analysis. Furthermore, causes of contingent heterogeneity (defined as the presence of statistical heterogeneity by chi-squared test with significance set at $\mathrm{P}$-value < 0.10 and measured by the quantities of heterogeneity by I2 (Higgins 2005, section 8.7.2)) were explored by comparing stratification between true ERAS protocols and other recovery strategies. An ERAS protocol was considered true when a minimal set of 7 ERAS interventions were used.

\section{R E S U L T S}

\section{Description of studies}

See: Characteristics of included studies; Characteristics of excluded studies; Characteristics of ongoing studies.

\section{Results of the search}

The systematic search was conducted in The Cochrane Library, Issue 1, 2009 (22 hits, 1 selected), The National Library of Medicine (MEDLINE) via PubMed (125 hits, 17 selected), Exerpta Medica via EMBASE (82 hits, 12 selected), ISI Web of Knowledge (135 hits, 14 selected), and web casts of the annual meetings of the American Society of Colon and Rectal Surgeons (ASCRS) (all published web cast until 2009, 0 selected). For detailed information about the search strategies and the numbers of hits we refer to additional Table 1. Altogether, the search resulted in 364 hits. After correction for duplicates, 241 publications remained.The first selection was performed based on the titles of publications and all clearly irrelevant publications were excluded. A total of 72 hits were considered possibly relevant based on their titles. The abstracts of these 72 publications were reviewed independently by two reviewers (WRS and JR). Differences between WRS and JR were discussed with CVL. A total of 55 publications could be rejected based on their abstracts. Eventually, 18 publications were selected for further evaluation and these are listed in this review with reasons for in- and exclusion. Also, 4 systematic reviews were identified (Wind 2006; Walter 2009; Gouvas 2009; Varadhan 2010) and included studies in these reviews, along with the reference lists were hand searched. No additional hits were identified.

\section{Included studies}

After completion of the search and complete review of the 18 selected remaining manuscripts, 6 RCTs were included in this review for all secondary analysis and background characteristics(see Characteristics of included studies). However, since 2 trials used insufficient number of ERAS items used in the protocol, only 4 RCT's were included in primary analysis.(Anderson 2003; Gatt 2005; Khoo 2007; Serclova 2009). The 6 trials included 452 patients, of whom 226 patients received ERAS treatment, and 226 patients received traditional care. Patient characteristics are depicted in Table 2. Inclusion criteria for all studies were similar; most included both benign and malignant disease. Majority of diagnosis consisted of malignant colon disease and inflammatory bowel diseases (i.e. Crohn's disease or ulcerative colitis), and included patients needed to be independently living at home. All studies only included elective surgery, and exclusion criteria were similar for most studies. Delaney et al, however, also included patients needing re-operations, including pelvic surgery. Baseline characteristics were similar for all studies. No significant differences in age and sex existed. The majority of included patients were classified as ASA 1 or 2 . Not all studies listed the ASA classification.

\section{Number of ERAS points used}

As mentioned, a complete peri-operative pathway according to ERAS principles includes 17 separate interventions (Lassen 2009). The actual number of interventions used differed greatly between included trials. Trials that were considered high quality used 11 or 12 of the 17 ERAS prespecified interventions versus 0 or 1 in the conventional group (pertaining epidural analgesia) (Anderson 2003; Gatt 2005; Serclova 2009). On the other hand, 2 trials incorporated only 4 or 6 ERAS items, while Muller also incorporated 4 ERAS items in the conventional group (Muller 2009).

Trials included for primary analysis

Since this clearly represents a bias on outcome analysis, primary 
analysis was performed on 4 RCTs incorporating no less than 7 items in the ERAS protocol and no more than 2 in the conventional group. To investigate effects when these trials were taken into account, a sensitivity analysis was performed, including aforementioned trials. Primary analysis resulted in a total inclusion for primary analysis of 4 RCT's, including 237 patients; 119 received ERAS treatment and 118 patients received conventional treatment.

\section{Indication}

Indications for resection are mentioned in the Included studies section. Indications were similar throughout the included studies and represent the normal clinical indications encountered during daily practice. Some also included pelvic surgery and/or rectal resections with TME. Whether these resections are fit for ERAS is unknown, so we found no reason to exclude these patients from analysis.

\section{Technique}

All randomised patients received segmental resection of colon, rectum and/or ileum by open surgical technique. Although inclusion criteria for this review also included laparoscopic techniques, no studies comparing conventional recuperation to laparoscopic technique with ERAS were identified.

\section{Trial designs}

All included trials can be classified as randomised controlled trials. Most were monocentric trials, except Muller 2009 (4 centres).

\section{Excluded studies}

From the selected 18, a total of 12 publications were excluded (see 'Excluded studies' table). Reasons for exclusion are mentioned in this table, mostly reasons for exclusion consisted of pseudo randomised studies and comparison of groups both receiving ERAS protocol treatment.

\section{Risk of bias in included studies}

The risk of bias for every included trial was assessed using the RevMan bias assessment tool and using the GRADE profile software. The under mentioned items were scored to assess bias and are depicted as methodological summary graph (Figure 2) and summary (Figure 3). Results are also depicted in the characteristics of included studies and Summary of findings for the main comparison.

Figure 2. Methodological quality graph: review authors' judgements about each methodological quality item presented as percentages across all included studies.

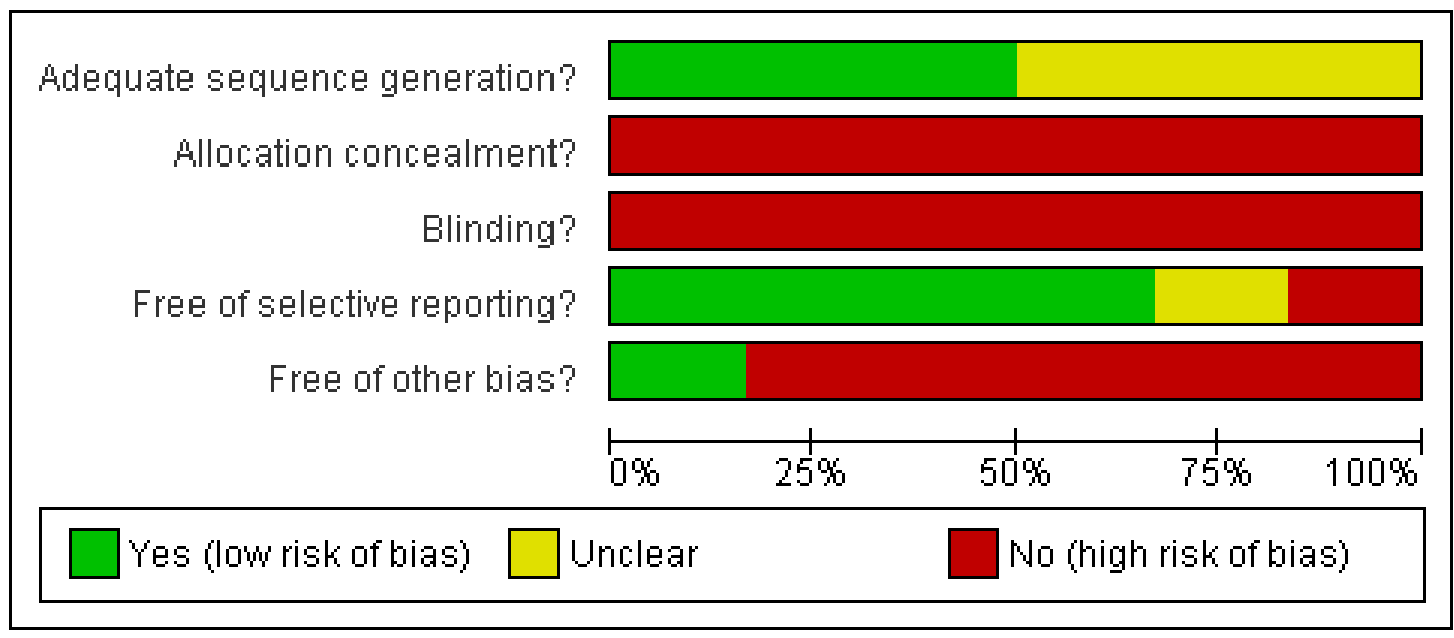

Fast track surgery versus conventional recovery strategies for colorectal surgery (Review) 
Figure 3. Methodological quality summary: review authors' judgements about each methodological quality item for each included study.

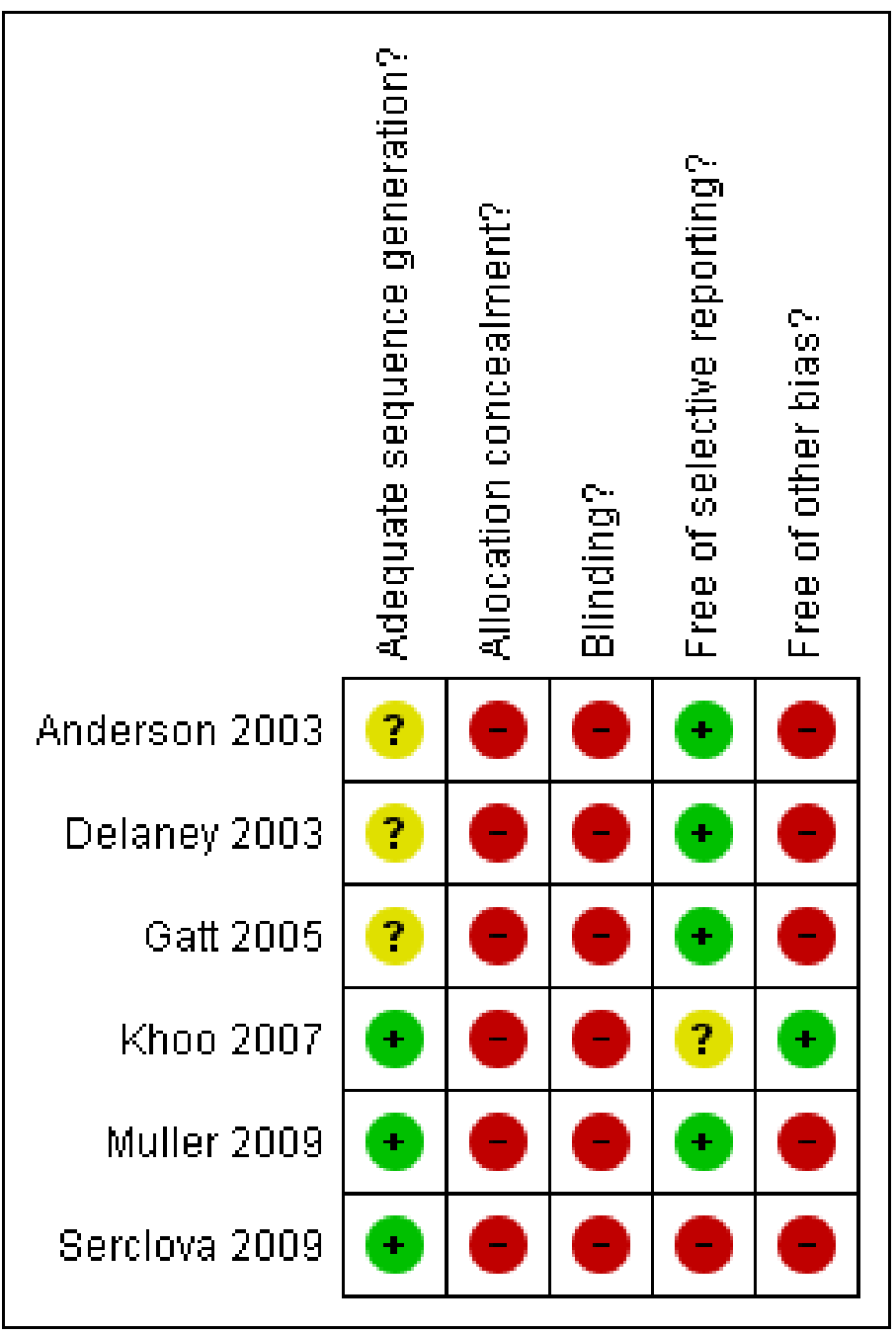

\section{Allocation}

Allocation sequences for included trials showed different risk for bias. Several trials used sealed envelopes for generating allocation, without reporting the exact sequence. It is known that the use of envelopes can result in bias, when care providers have an interest in allocating certain patients to the preferred treatment method.

\section{Blinding}

None of the included trials used blinding, nor for the patients nor for the treating surgeon. However, the investigated intervention (ERAS) has an intrinsic problem with blinding; an important intervention in ERAS is the pre-operative information provided to patients, which provides knowledge pertaining the operation and the expected peri- and postoperative course. Therefore, blinding patients is not possible. Blinding the surgeon is also restricted, because of the differences in surgical approach and postoperative care. However, blinding of outcome using an impartial outcome assessor is possible, but no adequate blinding was performed in included trials.

This may not be a problem, except when different surgeons are involved in caring for these patients and these surgeons have different experiences and preferences towards ERAS. This was the case in one trial (Delaney 2003) and this effect was also investigated. Analysis showed that ERAS surgeons caring for ERAS pa- 
tients actually showed better results (especially in length of stay) than traditional surgeons caring for ERAS patients. In fact, ERAS surgeons caring for conventional patients, also showed a shorter length of stay than conventional surgeons caring for conventional patients. Also, confounding could have been present by implementing both an ERAS care pathway and conventional care in the same hospital ward, presenting the risk of "cross contamination" by one protocol to another.

\section{Incomplete outcome data}

Most data that was described by authors as being recorded during trials are also reported. One outcome parameter that was insufficiently recorded was the effect of readmissions on hospital stay. Only 1 study (Khoo 2007) reported the length of stay associated with readmissions, however, only as absolute stay and this was not included in analysis.

\section{Selective reporting}

Data recorded was mostly reported, and therefore the risk of selective reporting seems to be small. However, a lot of data is reported as median with interquartile range, suggesting large outliers in the raw data. In the relative small populations studied, this could represent a bias, when these outliers occurred mostly in one of the groups.

\section{Other potential sources of bias}

Another potential source of bias in measuring the treatment effect of ERAS is the fact that in the conventional protocol, some of the ERAS interventions are also used. And since the exact contribution of the separate interventions in ERAS protocols are not known, the measured effect can be biased by these interventions used in the conventional protocol. In Figure 1, the number of interventions used in the ERAS protocols used in the included studies are shown. Publication bias was investigated using funnel plots (Figure 3). The risk of this bias can be considered as low, and therefore, no downgrading was performed on this item.

\section{Effects of interventions}

See: Summary of findings for the main comparison Primary analyses ERAS versus conventional for colorectal surgery

\section{Primary analysis}

As mentioned, primary analysis of pooled data was performed on 4 RCT's that were deemed of high(er) quality, because of the ratio of ERAS items used in their protocols (Anderson 2003; Gatt 2005; Khoo 2007; Serclova 2009).

\section{Primary outcome measures}

Mortality

Mortality after elective colorectal surgery is exceedingly rare. Mortality in included studies was $1(0.4 \%)$ in ERAS patients versus $3(1.3 \%)$ in conventional patients (Analysis 1.1, Figure 4), and showed no statistical difference between groups ((RR 0.53; $95 \%$ CI 0.12 to 2.38$)$ )

Figure 4. Forest plot of comparison: I Primary analyses ERAS versus conventional, outcome: I.I Mortality.

\begin{tabular}{|c|c|c|c|c|c|c|c|c|}
\hline \multirow[b]{2}{*}{ Study or Subgroup } & \multicolumn{2}{|c|}{ ERAS } & \multicolumn{2}{|c|}{ Cormentional } & \multirow[b]{2}{*}{ Weight } & \multirow{2}{*}{$\begin{array}{c}\text { Risk Ratio } \\
\text { M-H, Fixed, 95\% Cl }\end{array}$} & \multirow{2}{*}{\multicolumn{2}{|c|}{$\begin{array}{c}\text { Risk Ratio } \\
\text { M-H, Fixed, 95\% Cl }\end{array}$}} \\
\hline & Events & Total & Events & Total & & & & \\
\hline Anderson 2003 & 0 & 14 & 1 & 11 & $35.8 \%$ & $0.27[0.01,5.97]$ & \begin{tabular}{l|l}
-1 \\
-2
\end{tabular} & \\
\hline Gatt 2005 & 1 & 19 & 0 & 20 & $10.5 \%$ & $3.15[0.14,72.88]$ & & \\
\hline Khoo 2007 & 0 & 35 & 2 & 35 & $53.7 \%$ & $0.20[0.01,4.02]$ & $\longleftarrow$ & \\
\hline Serclova 2009 & 0 & 51 & 0 & 52 & & Not estimable & & \\
\hline Total $(95 \% \mathrm{Cl})$ & & 119 & & 118 & $100.0 \%$ & $0.53[0.12,2.38]$ & & \\
\hline Total events & 1 & & 3 & & & & & \\
\hline $\begin{array}{l}\text { Heterogeneity: } \mathrm{Chi}^{2} \\
\text { Test for overall effec }\end{array}$ & $\begin{array}{l}1.83, \mathrm{df}= \\
Z=0.82\end{array}$ & $\begin{array}{l}2(P= \\
P=0.4\end{array}$ & $\begin{array}{l}0.40) ; 1^{2}= \\
\text { 1) }\end{array}$ & & & & $\begin{array}{l}0.51 \\
\text { ERAS }\end{array}$ & $\begin{array}{lccc}1 & 5 & 5 & 10 \\
\text { Conventional }\end{array}$ \\
\hline
\end{tabular}


Complications

In the ERAS group 34 (28.5\%) patients sustained complications, while $67(56.8 \%)$ patients in the conventional group encountered complications (Analysis 1.2; Figure 5). The ERAS patients encountered significantly less complications (RR 0.52; 95\% CI 0.38 to 0.71$), \mathrm{p}<0.0001)$. Since not all complications represent the same negative risk for an intervention, complications were divided into major and minor complications, and where the exact complications were not explicated, they were deemed undefined.

Figure 5. Forest plot of comparison: I Primary analyses ERAS versus conventional, outcome: I.2 All complications.

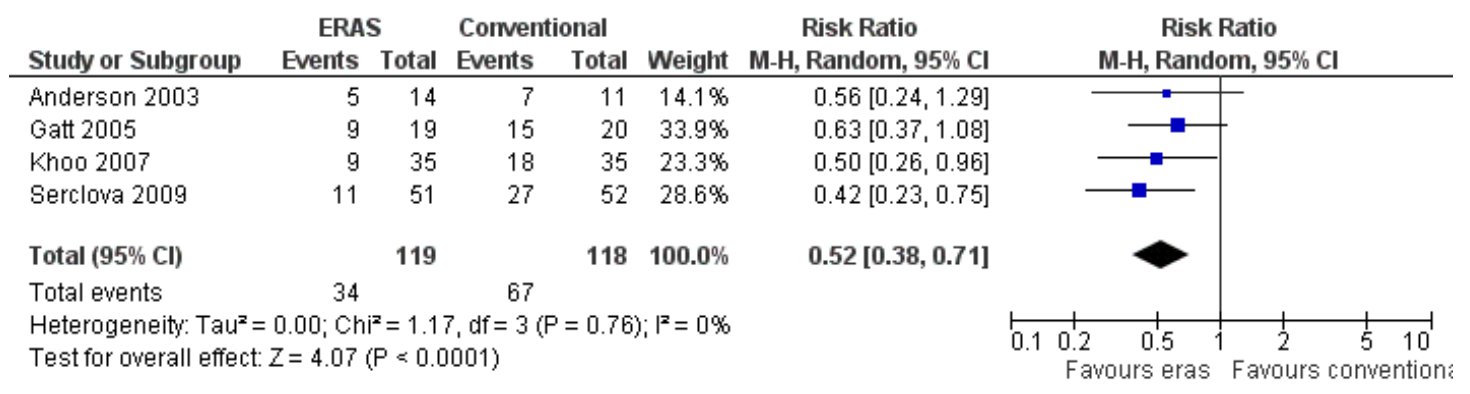

Major complications occurred in $6(8.8 \%)$ of ERAS patients and in $14(21.2 \%)$ of conventionally treated patients (RR $0.45 ; 95 \%$ CI 0.09 to 2.32, $\mathrm{p}=0.34$ ) (Analysis 1.3. Figure 6). These included mortality. In total, 17 (25\%) of ERAS patients endured minor complications versus $26(39.4 \%)$ of conventionally treated patients. (RR 0.63; 95\% CI 0.38 to 1.05 ) (Analysis 1.4,Figure 7), and 11 ERAS patients versus 7 conventional patients endured an undefined complication (described as no serious complications) (Analysis 1.5; Figure 8).

Figure 6. Forest plot of comparison: I Primary analyses ERAS versus conventional, outcome: I.3 Major Complications.

\begin{tabular}{|c|c|c|c|c|c|c|c|c|c|}
\hline \multirow[b]{2}{*}{ Study or Subgroup } & \multicolumn{2}{|c|}{ ERAS } & \multicolumn{2}{|c|}{ Cormentional } & \multirow[b]{2}{*}{ Weight } & Risk Ratio & \multirow{2}{*}{\multicolumn{2}{|c|}{$\begin{array}{c}\text { Risk Ratio } \\
\text { M-H, Random, } 95 \% \mathrm{Cl}\end{array}$}} & \\
\hline & Events & Total & Events & Total & & M-H, Random, $95 \%$ Cl & & & \\
\hline Anderson 2003 & 1 & 14 & 2 & 11 & $27.5 \%$ & $0.39[0.04,3.79]$ & $\Rightarrow$ & & \\
\hline Gatt 2005 & 4 & 19 & 3 & 20 & $41.5 \%$ & $1.40[0.36,5.46]$ & & & \\
\hline Khoo 2007 & 1 & 35 & 9 & 35 & $31.0 \%$ & $0.11[0.01,0.83]$ & $=$ & & \\
\hline Total $(95 \% \mathrm{Cl})$ & & 68 & & 66 & $100.0 \%$ & $0.45[0.09,2.32]$ & & & \\
\hline Total events & 6 & & 14 & & & & & & \\
\hline $\begin{array}{l}\text { Heterogeneity: } \mathrm{Tau}^{2} \text {, } \\
\text { Test for overall effect }\end{array}$ & $\begin{array}{l}1.20 ; \mathrm{Chi} \\
\mathrm{z}=0.95\end{array}$ & $\begin{array}{l}P=4.70 \\
P=0.3\end{array}$ & , df $=2(F$ & $=0.10)$ & $i^{2}=57 \%$ & & $\begin{array}{lll}0.1 & 0.2 & 0.5 \\
& \text { Favours ERAS }\end{array}$ & $\begin{array}{c}1 \\
\end{array}$ & $\begin{array}{c}510 \\
510 \\
\text { onventiona }\end{array}$ \\
\hline
\end{tabular}

Fast track surgery versus conventional recovery strategies for colorectal surgery (Review) 
Figure 7. Forest plot of comparison: I Primary analyses ERAS versus conventional, outcome: I.4 Minor complications.

\begin{tabular}{|c|c|c|c|c|c|c|c|c|}
\hline \multirow[b]{2}{*}{ Study or Subgroup } & \multicolumn{2}{|c|}{ ERAS } & \multicolumn{2}{|c|}{ Corventional } & \multirow[b]{2}{*}{ Weight } & \multirow{2}{*}{$\begin{array}{c}\text { Risk Ratio } \\
\text { M-H, Random, } 95 \% \mathrm{Cl}\end{array}$} & \multirow{2}{*}{\multicolumn{2}{|c|}{$\begin{array}{c}\text { Risk Ratio } \\
\text { M-H, Random, } 95 \% \mathrm{Cl}\end{array}$}} \\
\hline & Events & Total & Events & Total & & & & \\
\hline Anderson 2003 & 4 & 14 & 5 & 11 & $23.8 \%$ & $0.63[0.22,1.80]$ & & 7 \\
\hline Gatt 2005 & 5 & 19 & 12 & 20 & $37.9 \%$ & $0.44[0.19,1.01]$ & & \\
\hline Khoo 2007 & 8 & 35 & 9 & 35 & $38.3 \%$ & $0.89[0.39,2.04]$ & & \\
\hline Total $(95 \% \mathrm{Cl})$ & & 68 & & 66 & $100.0 \%$ & $0.63[0.37,1.05]$ & & \\
\hline Total events & 17 & & 26 & & & & & \\
\hline $\begin{array}{l}\text { Heterogeneity: Tau }{ }^{2} \\
\text { Test for overall effec }\end{array}$ & $\begin{array}{l}0.00 ; \text { Chi } \\
z=1.79\end{array}$ & $\begin{array}{l}P^{2}=1.3 \\
P=0.0\end{array}$ & $9, d f=2$ & $=0.50)$ & $; I^{2}=0 \%$ & & $\begin{array}{ccc} & 1 & 1 \\
0.1 & 0.2 & 0.5 \\
& \text { Favours ERAS }\end{array}$ & 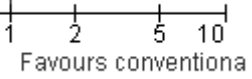 \\
\hline
\end{tabular}

Figure 8. Forest plot of comparison: I Primary analyses ERAS versus conventional, outcome: I.5 Undefined complications.

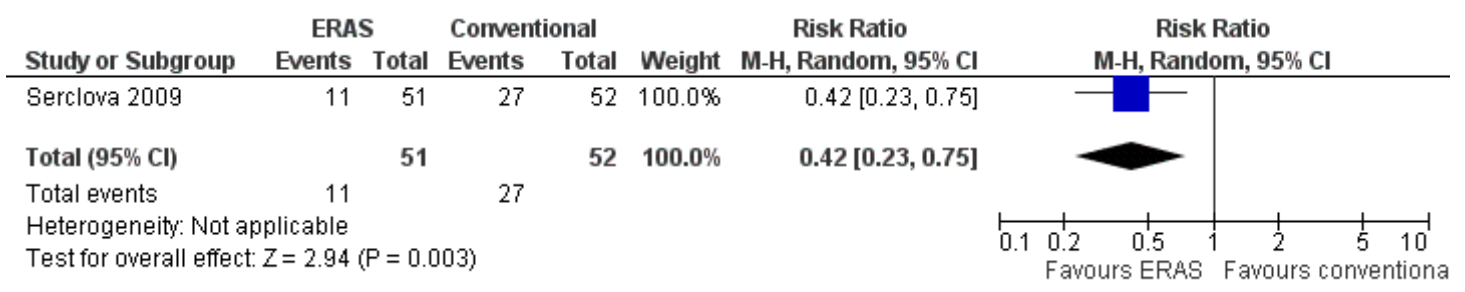

Readmissions

In total, 4 patients $(3.3 \%)$ from the ERAS study group and 5 patients $(4.2 \%)$ in the conventional group had to be readmitted. (RR 0.87; 95\% CI 0.08 to 9.39; Analysis 1.6, Figure 9) The effect on total hospital stay could not be clearly identified. However, one ERAS patient did have to be readmitted for 7 days (Khoo 2007), which could effect the analysis.

Figure 9. Forest plot of comparison: I Primary analyses ERAS versus conventional, outcome: I.6 Readmissions.

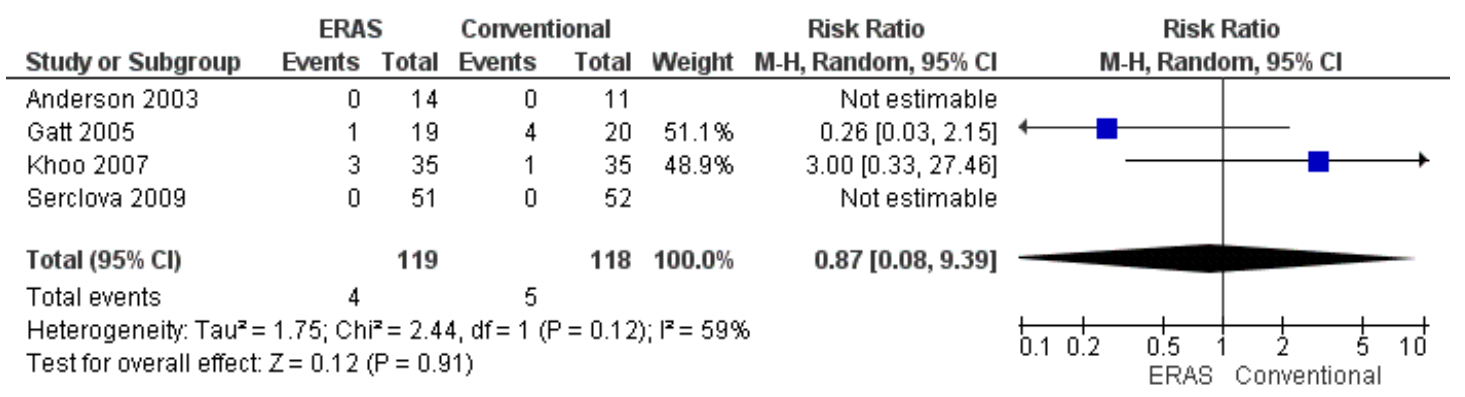

Fast track surgery versus conventional recovery strategies for colorectal surgery (Review) 
Length of hospital stay

Primary outcome measures were defined as length of hospital stay and complications, including readmissions. The total length of hospital stay was defined in included studies as the primary length of stay. Insufficient data on added length of stay due to readmissions was available. The primary length of stay (Analysis 1.7; Figure 10) was shorter for the ERAS treated patients (MD -2.94 days; $95 \%$ CI -3.69 to -2.19$)$. Since not all reasons for readmissions are reported, the effect on hospital stay is unknown.

Figure 10. Forest plot of comparison: I Primary analyses ERAS versus conventional, outcome: I.7 hospital stay [days].

\begin{tabular}{|c|c|c|c|c|c|c|c|c|c|c|}
\hline Study or Subgroup & $\begin{array}{r}E \\
\text { Mean [days] }\end{array}$ & $\begin{array}{l}\text { RAS } \\
\text { SD [days] }\end{array}$ & Total & \multicolumn{3}{|c|}{ Comventional } & \multicolumn{2}{|r|}{ Mean Difference } & \multicolumn{2}{|c|}{$\begin{array}{c}\text { Mean Difference } \\
\text { IV, Fixed, } 95 \% \mathrm{Cl} \text { [days] }\end{array}$} \\
\hline Gatt 2005 & 6.6 & 4.4 & 19 & 9 & 4.6 & 20 & $7.1 \%$ & $-2.40[-5.22,0.42]$ & & \\
\hline Total $(95 \% \mathrm{Cl})$ & & & 119 & & & 118 & $100.0 \%$ & $-2.94[-3.69,-2.19]$ & & \\
\hline $\begin{array}{l}\text { Heterogeneity: } \mathrm{Chi}^{2}= \\
\text { Test for overall effect }\end{array}$ & $\begin{array}{l}0.27, d f=3(P= \\
Z=7.65(P<0\end{array}$ & $\begin{array}{l}=0.97) ;\left.\right|^{2}=0 \\
.00001)\end{array}$ & & & & & & & $\begin{array}{cc}1 & 1 \\
-4 & -2 \\
& \mathrm{ER} t\end{array}$ & 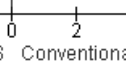 \\
\hline
\end{tabular}

\section{further reported outcome measures}

All studies reported a number of additional outcome measures. These were very heterogenic, and therefore not suitable for metaanalysis.

gastro-intestinal function

Four studies reported on gastro-intestinal function postoperatively. One trial (Gatt 2005) showed a significant shorter time to oral feeding for ERAS patients (48 vs $90 \mathrm{~h}, \mathrm{p} 0.042$ ), as did Khoo et al, with ERAS patients tolerating a solid diet on postoperative day 1 (range 0-6 days) against 4 days for the conventional group (range 2-9 days). The bowel function, defined as passing stool or functioning stoma, was also shorter for ERAS patients in this study; 3 days [1-5] for ERAS patients versus 5 [0-23] for conventionally treated patients $(\mathrm{p}<0.001)$. Anderson also reported an earlier return of gut function, defined as tolerating 3 light meals a day for ERAS patients (48[33-55]h vs 76[70-110)h, respectively), as did Serclova, with earlier bowel movement $(1.3 \pm 0.8$ vs $3.1 \pm 1.0$ days) and passing stool sooner $(2.1 \pm 1.1$ vs $3.9 \pm 1.1$ days $)$ for the ERAS treated patients. they also reported significantly more nausea in the conventional group on postoperative days 2-4, without an effect of the use of nasogastric tubes.

intravenous fluid administration

Analogue to earlier solid diet and normalisation of gastro-intestinal function, several trials also reported on the need for supplemental intravenous fluid. In the Anderson trial, iv fluids were discontinued at a median of 36 hours [24-37] in the ERAS group versus $57 \mathrm{~h}$ [42-105] in controls $(\mathrm{p}=0.001)$. Gatt found a similar effect with discontinuing iv fluids at median 33.2 hours for the
ERAS group vs $68 \mathrm{~h}$ for controls ( $\mathrm{p}=0.007$ ). Restriction of perioperative fluid administration is one of the ERAS working groups recommendations. However, only 2 of the included trials used this in their ERAS protocols. Muller did find in a secondary analysis that along with effective epidural analgesia, perioperative fluid restriction was an independent predictor for complications. intraoperative fluid administration was lower for ERAS patients here ( $1925 \mathrm{~mL}$ vs $2950 \mathrm{~mL}$ median, $\mathrm{p}<0.001)$

pain scores and analgesia

The use of analgesics varied greatly between study protocols. In some studies, epidural analgesia was used for all patients (Muller 2009; Khoo 2007; Gatt 2005; Serclova 2009), while one trial did not incorporate epidurals at all (Delaney 2003). The only trial that only used epidurals for ERAS patients (Anderson 2003) showed a significant increase in pain postoperatively in control patients on coughing, movement and at rest for the conventional group, while no increase was found for the ERAS group. Only by day 7, pain scores were similar between both groups, but pain on coughing remained significantly increased in controls. Delaney used morphine Patient Controlled Analgesia for all patients, that was changed for oral oxycodone on day 2 in ERAS patients and when control patients tolerated oral fluids. They found no differences in the amount of opiates used or in pain scores. Four studies that used epidural analgesics (EDA) in all patients, and 3 did not report postoperative pain scoring (Khoo 2007; Gatt 2005; Muller 2009). Muller did report similar effective EDA analgesia between both groups (79 vs $80 \%$ ), while Serclova at all reported the use of EDA 
in $100 \%$ of ERAS patients and $62 \%$ in controls. $14 \%$ of EDA in ERAS patients failed versus $28 \%$ in controls. VAS scores, both highest recorded per day and median, were significantly lower for ERAS patients between postop days 0-5 (p,0.001).

mobilisation

Trials that reported on postoperative mobilisation showed an overall better mobilisation for ERAS patients. Both mobilisation on postop day 0 (Serclova 2009) and day 1 (Gatt 2005) was better for ERAS patients. this did not result in better physiological parameters however, since FEV1 and FVC was significantly reduced in both ERAS and controls postoperatively and no difference between the groups was recorded (Gatt 2005). Other outcome measures reflecting improved preservation of physiology also showed no differences between both groups; hand grip strength showed no difference (Gatt 2005) and quality of life scores also showed no differences (Delaney 2003).

duration of surgery

Duration of surgery was only reported in one trial (Muller 2009), and showed no difference in median duration of surgery (140 vs 120 minutes). However, the incision used was also the same for both study arms.

other outcome measures
No studies investigated cost effectiveness of ERAS protocols, nor was any mention on the influence of ERAS on quality of life found. Also, no study investigated ERAS failure, i.e. individual patients within an ERAS program displaying failure to thrive. Moreover, no study reported on the compliance of individual ERAS items within their study protocols, while lack of compliance will inevitably lead to bias in data provided.

\section{sensitivity analysis}

As mentioned, 2 of the included trials used a very limited number of ERAS items. When we did include these trials in analysis, these 6 RCT's included 452 patients, 226 patients per study group. The mortality analysis did not differ, because neither of the 2 trials reported mortality.

\section{Complications}

The total number of complications for the entire population was 54 for the ERAS group versus 105 for conventional patients (RR 0.51 ; $95 \%$ CI 0.39 to 0.67 )(Analysis 2.2; Figure 11). This was further subdivided into major complications; 14 for ERAS patients versus 28 in conventional patients (RR $0.50 ; 95 \%$ CI 0.28 to 0.92 )(Analysis 2.3, Figure 12) and minor complications; 29 for ERAS patients versus 50 for conventional groups (RR 0.57; $95 \%$ CI 0.38 to 0.85 ) (Analysis 2.4; Figure 13, Figure 14).

Figure I I. Forest plot of comparison: 2 Sensitivity analyses ERAS vs conventional, outcome: 2.2 Total complications.

\begin{tabular}{|c|c|c|c|c|c|c|c|c|}
\hline \multirow[b]{2}{*}{ Study or Subgroup } & \multicolumn{2}{|c|}{ ERAS } & \multicolumn{2}{|c|}{ corventional } & \multirow[b]{2}{*}{ Weight } & $\begin{array}{c}\text { Risk Ratio } \\
\text { M-H.Fixed. 95\% Cl }\end{array}$ & \multirow{2}{*}{\multicolumn{2}{|c|}{$\begin{array}{c}\text { Risk Ratio } \\
\text { M-H, Fixed, 95\% Cl }\end{array}$}} \\
\hline & Events & Total & Events & Total & & M-H, Fixed, 95\% Cl & & \\
\hline Anderson 2003 & 5 & 14 & 7 & 11 & $7.5 \%$ & $0.56[0.24,1.29]$ & & \\
\hline Delaney 2003 & 7 & 31 & 10 & 33 & $9.2 \%$ & $0.75[0.32,1.71]$ & $\rightarrow$ & \\
\hline Gatt 2005 & 9 & 19 & 15 & 20 & $13.9 \%$ & $0.63[0.37,1.08]$ & $\rightarrow$ & \\
\hline Khoo 2007 & 9 & 35 & 18 & 35 & $17.1 \%$ & $0.50[0.26,0.96]$ & $\rightarrow-$ & \\
\hline Muller 2009 & 13 & 76 & 28 & 75 & $26.8 \%$ & $0.46[0.26,0.81]$ & $\rightarrow-$ & \\
\hline Serclova 2009 & 11 & 51 & 27 & 52 & $25.4 \%$ & $0.42[0.23,0.75]$ & $\rightarrow-$ & \\
\hline Total $(95 \% \mathrm{Cl})$ & & 226 & & 226 & $100.0 \%$ & $0.51[0.39,0.67]$ & 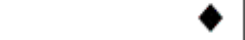 & \\
\hline Total events & 54 & & 105 & & & & & \\
\hline $\begin{array}{l}\text { Heterogeneity: } \mathrm{Chi}^{2} \\
\text { Test for overall effec }\end{array}$ & $\begin{array}{l}2.05, \mathrm{df}= \\
Z=4.92\end{array}$ & $\begin{array}{l}5(P= \\
P<0.0\end{array}$ & $\begin{array}{l}0.84) ;\left.\right|^{2}= \\
0001)\end{array}$ & & & & $\begin{array}{cc}0.01 & 0.1 \\
\text { Favours experimental }\end{array}$ & $\begin{array}{cc}10 & 100 \\
\text { Favours control }\end{array}$ \\
\hline
\end{tabular}

Fast track surgery versus conventional recovery strategies for colorectal surgery (Review) 
Figure 12. Forest plot of comparison: 2 Sensitivity analyses ERAS vs conventional, outcome: 2.3 Major complications.

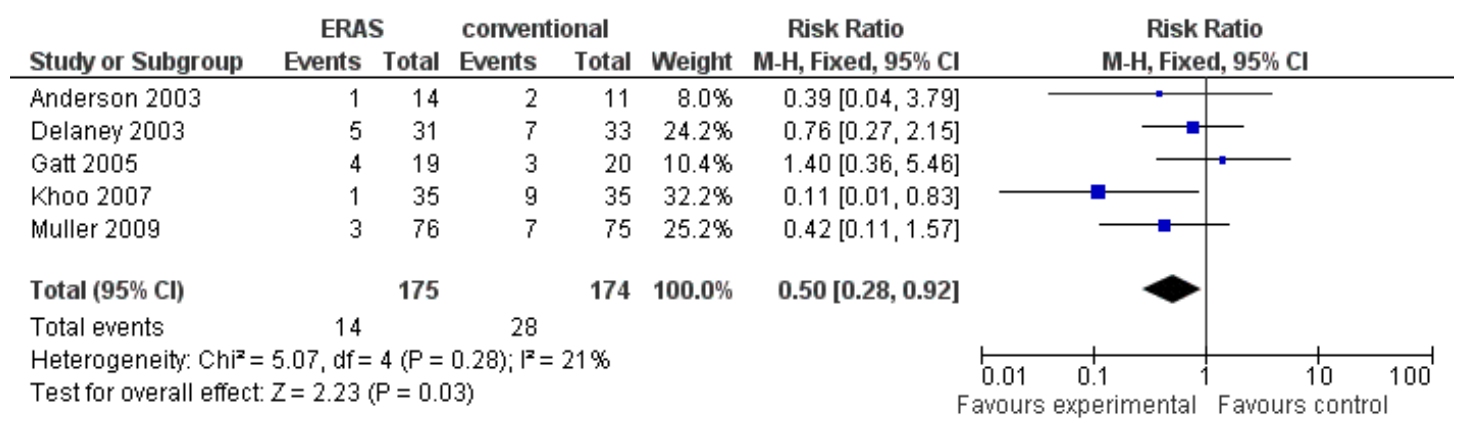

Figure 13. Forest plot of comparison: 2 Sensitivity analyses ERAS vs conventional, outcome: 2.5 Minor complications.

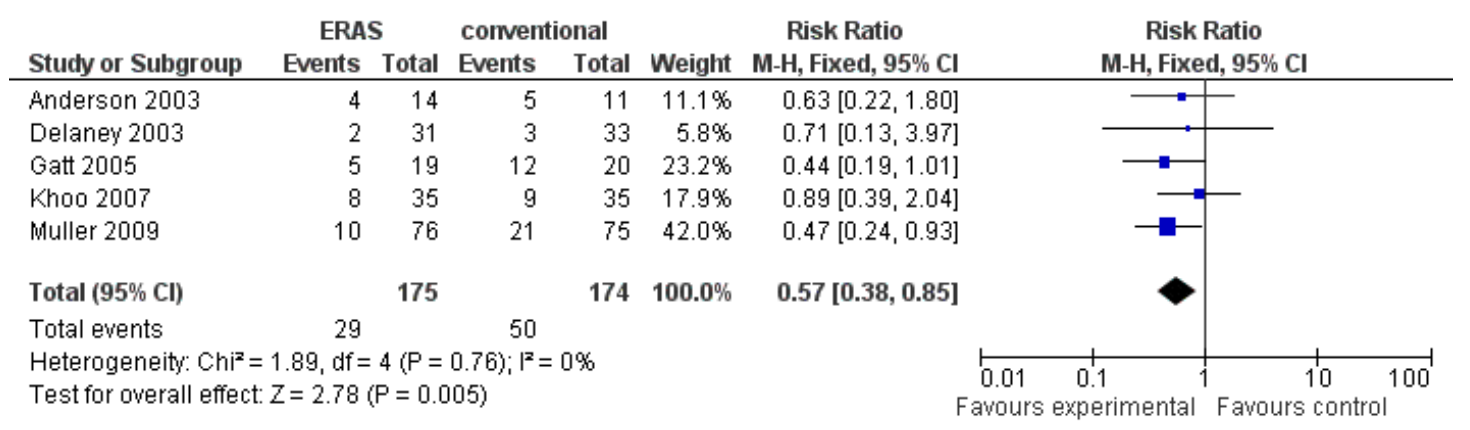

Figure 14. Forest plot of comparison: 2 Sensitivity analyses ERAS vs conventional, outcome: 2.5 Undefined complications.

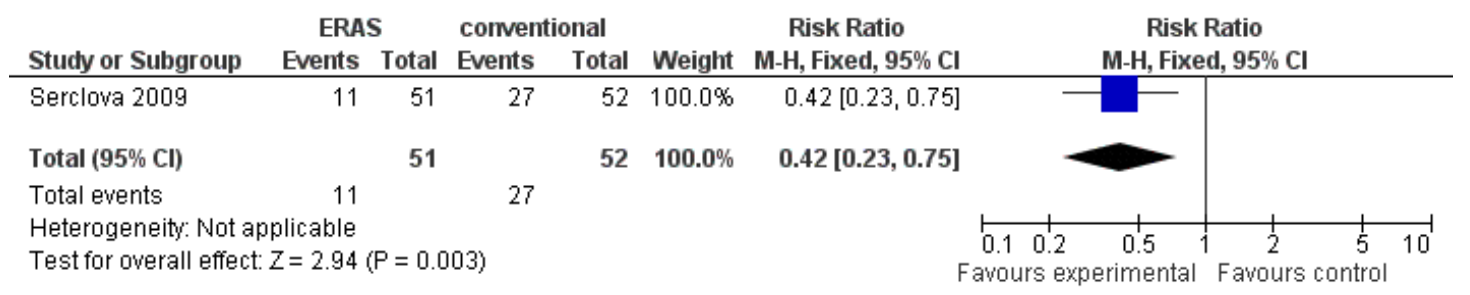

\section{Readmissions}

The number of readmissions showed no significant differences between both groups (RR 0.79; 95\% CI 0.36 to 1.76) (Analysis
2.6; Figure 15). In total, 10 ERAS patients had to be readmitted against 13 conventionally treated patients. There were now 10 readmissions in the ERAS population and 13 in the conventional 
Figure 15. Forest plot of comparison: 2 Sensitivity analyses ERAS vs conventional, outcome: 2.3 readmissions.

\begin{tabular}{|c|c|c|c|c|c|c|c|c|c|}
\hline \multirow[b]{2}{*}{ Study or Subgroup } & \multicolumn{2}{|c|}{ ERAS } & \multicolumn{2}{|c|}{ corventional } & \multirow[b]{2}{*}{ Weight } & \multirow{2}{*}{$\begin{array}{c}\text { Risk Ratio } \\
\text { M-H, Fixed, } 95 \% \mathrm{Cl}\end{array}$} & \multirow{2}{*}{\multicolumn{3}{|c|}{$\begin{array}{c}\text { Risk Ratio } \\
\text { M-H, Fixed, 95\% Cl }\end{array}$}} \\
\hline & Events & Total & Events & Total & & & & & \\
\hline Anderson 2003 & 0 & 14 & 0 & 11 & & Not estimable & & & \\
\hline Delaney 2003 & 3 & 31 & 6 & 33 & $45.7 \%$ & $0.53[0.15,1.95]$ & & & \\
\hline Gatt 2005 & 1 & 19 & 4 & 20 & $30.6 \%$ & $0.26[0.03,2.15]$ & & & \\
\hline Khoo 2007 & 3 & 35 & 1 & 35 & $7.9 \%$ & $3.00[0.33,27.46]$ & & & \\
\hline Muller 2009 & 3 & 76 & 2 & 75 & $15.8 \%$ & $1.48[0.25,8.61]$ & & & \\
\hline Serclova 2009 & 0 & 51 & 0 & 52 & & Not estimable & & & \\
\hline Total $(95 \% \mathrm{Cl})$ & & 226 & & 226 & $100.0 \%$ & $0.79[0.36,1.76]$ & & & \\
\hline Total events & 10 & & 13 & & & & & & \\
\hline $\begin{array}{l}\text { Heterogeneity: Chi } \\
\text { Test for overall effec }\end{array}$ & $\begin{array}{l}3.29, \mathrm{df}= \\
Z=0.57\end{array}$ & $\begin{array}{l}3(\mathrm{P}= \\
\mathrm{P}=0.5\end{array}$ & $\begin{array}{l}0.35 ; 1^{2}= \\
7)\end{array}$ & & & & 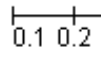 & $\begin{array}{l}0.51 \\
\text { ERAS }\end{array}$ & 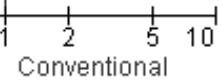 \\
\hline
\end{tabular}

Hospital stay

The length of hospital stay for all trials was shorter in sensitivity analysis; MD -2.51 days; $95 \%$ CI -3.54 to -1.47 , which was significant $(\mathrm{p}<0.00001)$ (Analysis 2.7; Figure 16).

Figure 16. Forest plot of comparison: 2 Sensitivity analyses ERAS vs conventional, outcome: 2.4 hospital stay [days].

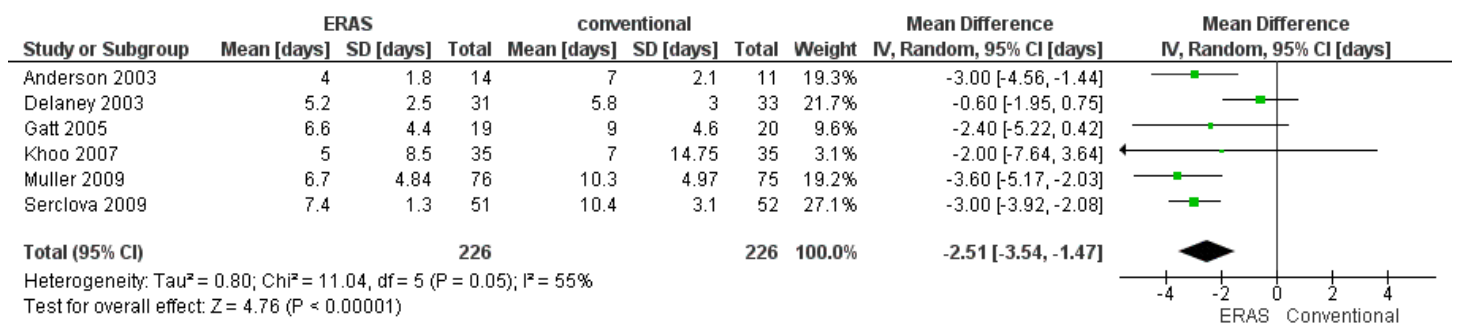

\section{ISCUSSION}

\section{Summary of main results}

This review searched for any RCT comparing conventional recovery strategies to ERAS recovery strategies after ileo-colorectal surgery. In all 17 RCT's were identified, and after applying selection criteria, 6 RCT's were included. Primary analysis was performed on the 4 RCT's who fulfilled the preset criteria for ERAS and conventional recovery strategies, and a sensitivity analysis on all 6 studies was performed. The 4 studies included 237 patients, 119 receiving ERAS and 118 receiving conventional treatment, whereas the 6 RCT included 452 patients, with 226 patients in both groups.

\section{Primary outcome measures}

As our most important outcome parameter, reflecting safety of the intervention, complications, including mortality was analysed. The total number of complications was relatively large in both 
groups. ERAS patients developed significantly less complications overall (RR 0.52 ; $95 \%$ CI 0.38 to $0.71, \mathrm{p}<0.0001$ ). When divided into major and minor complications, however, no significant difference in major (RR 0.45 ; $95 \%$ CI 0.09 to 2.32 , $\mathrm{p}=0.34$ ) or minor (RR 0.63 ; $95 \%$ CI 0.38 to $1.05, \mathrm{p}=0.07$ ) complications was found. These analyses suggest that ERAS is a safe treatment protocol and does not lead to more complications. When lesser quality studies were added to the analysis, the effect remained the same. However, there was heterogeneity in this analysis, especially in combined complications. The way complications were recorded was not constant between studies, nor were definitions of complications. Also, complications were scored in hospital treatment, and since the overall hospital stay differed between studies (partly because of types of surgery included) it is unclear whether all complications were recorded. It is nonetheless not likely that major complications would not be recorded, even when presenting after discharge.

The risk of readmissions was not increased with ERAS patients, while the primary length of hospital stay was shorter in ERAS treated patients (MD -2.94 days; 95\% CI -3.69 to -2.19 ). However, insufficient data concerning the effect of readmission on total length of stay was reported, so total length of hospital stay could not be analysed. But since the number of readmissions is not different between ERAS and conventional groups, the effect would be minimal.

Length of hospital stay was significantly reduced in the ERAS group. However, since a primary goal of the intervention is reducing hospital stay, bias may have occurred in this outcome parameter. Additionally, we do not feel that hospital stay is a medically important outcome parameter. This in accordance with GRADE methodology, as presented by Guyatt (Guyatt 2008), in which the importance of an outcome to patients is put as the central perspective to establish importance of outcome parameters. Other secondary outcome parameters were divers, and no meta analyses could be performed. However, reported outcomes like return of gastrointestinal function and pain, seem to favour the ERAS protocol as well.

The results therefore seem to advocate the use of an ERAS protocol as standard care. More large trials with more quality control can provide more power and may prove ERAS to be superior to conventional care.

The use of analgesics and especially the use of epidural analgesia was studied in two trials, with contradictory results. Anderson 2003 used EDA for ERAS patients, and PCA morphine for controls. This study showed excellent analgesia in ERAS patients, with no significant differences in pain levels before and after surgery. Pain was however significantly higher for controls, an effect which continued to day 7 postoperatively, suggesting a superiority in using EDA. However, Serclova 2009, besides using standard epidurals in ERAS patients, also used EDA in $68 \%$ of control patients and still reported significant lower pain scores in the ERAS group. However, failure rates were higher in the conventional EDA's, and no analysis between both subgroups with effective epidurals was made.

\section{Overall completeness and applicability of evidence}

Several outcome parameters possibly aiding in an advice on implementation of the ERAS protocol are not available in literature. Mostly, long term outcome parameters like oncological survival, quality of life after surgery are not investigated yet. Also, economical effects of the intervention have not been investigated prospectively, although retrospective evidence on cost effectiveness is now available (Sammour 2010). Measures like cost effectiveness and implementation costs have not been explored.

Furthermore, evidence on the effects of ERAS with different operative techniques like laparoscopy have not been analysed. The combination of ERAS and laparoscopy could make a difference in primary outcome parameters. Also, all included studies focus on the differences between "full" ERAS programs and conventional recovery strategies. The effect of separate interventions in the ERAS protocol have not been independently studied. This analysis is nonetheless very important, because the exact value of the separate interventions is not known, so further optimisation of ERAS protocols is more difficult. Exact knowledge on separate effects could also aid in making ERAS programs more (cost-) efficient and effective.

\section{Quality of the evidence}

All included studies were classified as Randomised Controlled Trials. However, methodological quality was not high (Summary of findings for the main comparison). None of the included trials used allocation concealment or blinding. This is hard to achieve in trials concerning complex interventions and surgical research. However, it may introduce bias that influences results. One trial proved this, by analysing the effect on hospital stay in comparing results by type of surgeon; they had both traditional surgeons and an ERAS surgeons caring for analysed patients. Analysis showed that a traditional surgeon caring for ERAS patients had longer lengths of stay than an ERAS surgeon, while ERAS surgeons treating conventional groups actually discharged patients earlier. This suggests that effects on length of stay between groups, could actually be caused by the mind set of staff, rather than the patients. All studies used length of hospital stay as the primary research question, implying this is a medically important parameter and reflects quality of recovery. No proof of this hypothesis exists however, and since primary research questions influence the study protocol this may produce bias in secondary outcome measures, as defined as complications. We feel this should be the most important primary outcome parameter, since this is the only quantative measure of safety and the primary goal of an intervention-comparison. Most studies used a power analysis for their inclusion, 
calculating expected effect on the length of stay parameter, thereby not calculating the number of patients needed to adequately reach significance on the null hypothesis for equality in complications. The sequence generation progress was also deemed unclear in most included studies, since most used an envelope method, which is prone to abuse. Selection bias is however less likely, because of contemporary groups and in some instances even "worse" patients (based on age, ASA and resections) in the ERAS group.

Another major problem with the evidence was identified however. None of the studies adequately reported on compliance with protocols or actions to prevent mixing of protocols (cross contamination). Therefore, the measured effects might easily be under- or overestimated. Moreover, it makes it less likely to identify elements in the ERAS protocols that are more or less important in reaching the goals of ERAS. We feel that in these studies, since some of the ERAS items were in fact used in conventional protocols, these effects would sooner be underestimated and that stringent monitoring of protocol compliance and explicit separation between conventional therapy and a full ERAS package could result in better outcome.

\section{AUTHORS, CONCLUSIONS}

\section{Implications for practice}

The use of ERAS programs in peri-operative care for abdominal (ileo-) colorectal surgery seems safe. Implementation in the identified RCT's showed a reduction in overall complications in the ERAS group, while procuring a decrease in hospital length of stay. However, major complications were not reduced, and the effect was due to a reduction in undefined complications in a single trial. More large studies, with more stringent quality criteria may improve power and provide proof of reducing complications. In the field, a common idea is although releasing patients earlier, ERAS leads to more readmissions. Although patients were released earlier, surprisingly no increase in readmissions have been observed. This while an 30 day follow up period was used, so it is unlikely any complications as reason for readmission were missed.
Although not statistically significant, It seems, however, that ERAS does decrease complications. However, available data does not provide proof that ERAS is superior to conventional technique and more evidence should be provided, as well as the quality of performed trials should be higher in order to adequately advocate using ERAS as the golden standard of care.

Available data does not provide insight into the effect of protocol adherence or implementation follow up. Simply implementing an ERAS protocol does not ensure results as found in this meta analysis; stringent overseeing of protocol adherence by all staff, as well as continued alertness for decreasing compliance also seems necessary, as many colleagues involved in ERAS implementations have found.

The results of this review are focused on relatively healthy patients, as most included subjects were ASA 1 or 2 . No data exists, therefore, to guarantee safety of implementing an ERAS protocol in a population with extended co-morbidity or higher age.

\section{Implications for research}

The number of studies found was relatively low, and aforementioned quality issues may bias results significantly. Therefore more large trials with better separation between conventional and ERAS protocols and monitoring of protocol compliance seems necessary. Furthermore, in light of current evidence, we feel ERAS should not be considered the new standard of care (yet).

Long term data on outcome, as well as important other factors in making a decision for an intervention, are also lacking. Quality of life data and data on physiological performance after 30 days have never been described, nor have data on cost-effectiveness or economic evaluations of ERAS programs. These parameters could play an important part in recommending ERAS treatment in colorectal surgery.

We have identified a large ongoing RCT (Wind 2006) and one prospective cohort study (Reurings 2009) that should provide more data in trials that more stringently separate intervention and control groups.

\section{R E F E R E N C E S}

\section{References to studies included in this review}

Anderson 2003 \{published data only\}

* Anderson, A.D, C.E McNaught, et al.Randomized

clinical trial of multimodal optimization and standard perioperative surgical care. Br J Surg 2003;90(12): 1497-504. [PUBMED: Anderson, A.D, C.E McNaught, et al.. Randomized clinical trial of multimodal optimization and standard perioperative surgical care. Br J Surg 2003;90 (12):1497-504.]
Delaney 2003 \{published data only\}

* Delaney, C.P, M. Zutshi, et al.Prospective, randomized, controlled trial between a pathway of controlled rehabilitation with early ambulation and diet and traditional postoperative care after laparotomy and intestinal resection. Dis Colon Rectum 2003;46(7):851-9.

Gatt 2005 \{published data only\}

* Gatt, M, A.D.G Anderson, et al.Randomized clinical trial of multimodal optimization of surgical care in patients 
undergoing major colonic resection. Br J Surg 2005;92(11): 1354-1362.

Khoo 2007 \{published data only\}

${ }^{*}$ Khoo, C. K.Vickery, C. J, et al.A prospective randomized controlled trial of multimodal perioperative management protocol in patients undergoing elective colorectal resection for cancer. Ann Surg 2007;245(6):867-72.

Muller 2009 \{published data only\}

* Muller, S, Zalunardo, M. P, et al.A fast-track program reduces complications and length of hospital stay after open colonic surgery. Gastroenterology 2009;136(3):842-7.

Serclova 2009 \{published data only\}

Zuzana serclova, Petr Dytrych, Jaroslav Marvan, et. al. Fast Track in open intestinal surgery: Prospective randomized study (Clinical Trials Gov Identifier no. NCT00123456).. Clinical Nutrition 2009;28:618-24.

\section{References to studies excluded from this review}

Basse 2005 \{published data only\}

* Basse, L.Jakobsen, D. H.Bardram, L.Billesbolle, P.Lund, C.Mogensen, T.Rosenberg, J.Kehlet, H. Functional recovery after open versus laparoscopic colonic resection: a randomized, blinded study. Ann Surg 2005;241(3):416-23.

Holte 2007 \{published data only\}

* Holte, K.Foss, N. B.Andersen, J.Valentiner, L.Lund, C.Bie, P.Kehlet, H. Liberal or restrictive fluid administration in fast-track colonic surgery: a randomized, double-blind study. Br J Anaesth 2007;99(4):500-8.

King 2006 \{published data only\}

* King, P. M.Blazeby, J. M.Ewings, P.Franks, P. J.Longman, R. J.Kendrick, A. H.Kipling, R. M.Kennedy, R. H. Randomized clinical trial comparing laparoscopic and open surgery for colorectal cancer within an enhanced recovery programme. Br J Surg 2006;93(3):300-8.

King 2008 \{published data only\}

* King, P. M.Blazeby, J. M.Ewings, P.Kennedy, R. H. Detailed evaluation of functional recovery following laparoscopic or open surgery for colorectal cancer within an enhanced recovery programme. Int J Colorectal Dis 2008;23 (8):795-800.

Kuzma 2008 \{published data only\}

* Kuzma, J. Randomized clinical trial to compare the length of hospital stay and morbidity for early feeding with opioid sparing analgesia versus traditional care after open appendectomy. Clinical Nutrition 2008;27(5):694-99.

Liu 2008 \{published data only\}

* Liu, Z.Wang, X. D.Li, L. [Perioperative fast track programs enhance the postoperative recovery after rectal carcinoma resection]. Zhonghua Wei Chang Wai Ke Za Zhi 2008;11(6):551-3.

MacKay 2006 \{published data only\}

* MacKay, G.Ihedioha, U.McConnachie, A.Serpell, M.Molloy, R. G.O'Dwyer, P. J. Laparoscopic colonic resection in fast-track patients does not enhance short-term recovery after elective surgery. Colorectal Dis 2007;9(4):

368-72.

Noblett 2006 \{published data only\}

* Noblett, S. E.Watson, D. S.Huong, H.Davison,

B.Hainsworth, P. J.Horgan, A. F. Pre-operative oral carbohydrate loading in colorectal surgery: a randomized controlled trial. Colorectal Disease 2006;8(7):563-569.

Raue 2004 \{published data only\}

* Raue, W.Haase, O.Junghans, T.Scharfenberg,

M.Muller, J. M.Schwenk, W. 'Fast-track' multimodal rehabilitation program improves outcome after laparoscopic sigmoidectomy: a controlled prospective evaluation. Surg Endosc 2004;18(10):1463-8.

Xu 2007 \{published data only\}

* Xu, J. M.Zhong, Y. S.Zhu, D. X.Ren, L.Wei, Y.Xue, Z. G.Jin, L.Niu, W. X.Qin, X. Y.Wu, Z. G. [Application of the protocol of enhanced recovery after surgery in colorectal surgery]. Zhonghua Wei Chang Wai Ke Za Zhi 2007;10(3): $238-44$.

Zutshi 2004 \{published data only\}

* Zutshi, M.Delaney, C. P.Senagore, A. J.Fazio, V. W. Shorter hospital stay associated with fastrack postoperative care pathways and laparoscopic intestinal resection are not associated with increased physical activity. Colorectal Dis 2004;6(6):477-80.

Zutshi 2005 \{published data only\}

* Zutshi, M.Delaney, C. P.Senagore, A. J.Mekhail, N.Lewis, B.Connor, J. T.Fazio, V. W. Randomized controlled trial comparing the controlled rehabilitation with early ambulation and diet pathway versus the controlled rehabilitation with early ambulation and diet with preemptive epidural anesthesia/analgesia after laparotomy and intestinal resection. Am J Surg 2005;189(3):268-72.

\section{References to ongoing studies}

\section{Reurings 2009 \{unpublished data only\}}

* Jurrian C Reurings, Willem R Spanjersberg, Henk JM Oostvogel, E Buskens, John Maring, Flip Kruyt, Camiel Rosman, Peter van Duivendijk, Cees HC Dejong, Cees JHM van Laarhoven. A prospective cohort study to investigate cost-minimisation, of Traditional open, open fAst track recovery and laParoscopic fASt track multimodal management, for surgical patients with colon carcinomas. (TAPAS study). BMC Surgery submitted.

Wind 2006 \{unpublished data only\}

Perioperative strategy in colonic surgery; LAparoscopy and/ or FAst track multimodal management versus standard care (LAFA trial). Ongoing study Starting date of trial not provided. Contact author for more information.

\section{Additional references}

\section{Anderson 2003}

Anderson, A. D.McNaught, C. E.MacFie, J.Tring, I.Barker, P.Mitchell, C. J. Randomized clinical trial of multimodal optimization and standard perioperative surgical care. $\mathrm{BrJ}$ Surg December 2003;90(12):1497-504. 


\section{Assmann 2000}

Assmann SF, Pocock SJ, Enos LE, Kasten LE. Subgroup analysis and other (mis)uses of baseline data in clinical trials. Lancet 2000;355(9209):1064-9.

\section{Basse 2000}

Basse, L.Hjort Jakobsen, D.Billesbolle, P.Werner, M.Kehlet, H. A clinical pathway to accelerate recovery after colonic resection. Ann Surg July 2000;232(1):51-7.

Basse 2002

L. Basse, H. H. Raskov, D. Hjort Jakobsen, E. Sonne, P. Billesbolle, H. W. Hendel, J. Rosenberg, H. Kehlets. Accelerated postoperative recovery programme after colonic resection improves physical performance, pulmonary function and body composition. Br J Surg April 2002;89 (4):446-53.

\section{Basse 2004}

Basse, L.Thorbol, J. E.Lossl, K.Kehlet, H. Colonic surgery with accelerated rehabilitation or conventional care. Dis Colon Rectum March 2004;47(3):221-7.

\section{Begg 1994}

Begg CB, Mazumdar M. Operating characteristics of a rank correlation test for publication bias.. Biometrics 1994;50(4): 1088-101

\section{Demets 1987}

Demets, D. L. Methods for combining randomized clinical trials: strengths and limitations. Stat Med Apr-May 1987;6 (3):341-50.

\section{Dersimonian 1986}

DerSimonian, R.Laird, N. Meta-analysis in clinical trials. Control Clin Trials Sep 1986;7(3):177-88.

\section{Egger 1997}

Egger M, Davey Smith G, Schneider M, Minder C. Bias in meta-analysis detected by a simple graphical test.. $B M J$ 1997;315(7109):629-34.

Gouvas 2009

Gouvas, N.Tan, E.Windsor, A.Xynos, E.Tekkis, P. P. Fasttrack vs standard care in colorectal surgery: a meta-analysis update. Int J Colorectal Dis 2009;24(10):1119-31.

\section{Guyatt 2008}

Guyatt, G.H. Oxman D.A, Vist, G.E. Kunz, R, et al.GRADE: what is "quality of evidence" and why is it important to physicians. BMJ 3 May 2008;336:995-8.

Hazebroek 2002

Hazebroek EJ, Color Study Group. COLOR: a randomized clinical trial comparing laparoscopic and open resection for colon cancer.. Surg Endosc 2002 Jun;16(6):949-53.

\section{Higgins 2002}

Higgins JPT, Thompson SG. Quantifying heterogeneity in a meta-analysis.. Statistics in Medicine 2002;21:1539-58.

\section{Higgins 2008}

Higgins JPT, Green S, editors. Cochrane Handbook for Systematic Reviews of Interventions 5.0.1 [updated September 2008]. http://www.cochrane-handbook.org/.

\section{Hozo 2005}

Hozo SP, Djulbegovic B, Hozo I. Estimating the mean and variance from the median, range, and the size of a sample.. BMC Medical Research Methodology 2005;5(1):13.

Kehlet 2003

Kehlet, H.Dahl, J. B. Anaesthesia, surgery, and challenges in postoperative recovery. Lancet Dec 6, 2003;362(9399): 1921-8.

Kehlet 2007

Kehlet, H. Epidural analgesia and recovery after abdominal surgery. Am J Surg February 2007;193(2):291.

Kehlet 2008

Kehlet, H. Fast-track colorectal surgery. Lancet March 8 2008;371(9615):791-3.

Keus 2009

Keus F, Wetterslev J, Gluud C, Gooszen HG, van Laarhoven CJ. Robustness assessments are needed to reduce bias in meta-analyses that include zero-event randomized trials. Am J Gastroenterol 2009 Mar;104(3):546-51.

Khoo 2007

Khoo, C. K.Vickery, C. J.Forsyth, N.Vinall, N. S.EyreBrook, I. A. A prospective randomized controlled trial of multimodal perioperative management protocol in patients undergoing elective colorectal resection for cancer. Ann Surg June 2007;245(6):867-72.

Kjaergard 2001

Kjaergard LL, Villumsen J, Gluud C. Reported methodological quality and discrepancies between large and small randomised trials in meta-analyses.. Annals of Internal Medicine 2001;135(11):982-9.

Lassen 2009

Lassen K, Soop M, Nygren J, Cox P.B, Henry P.O, Spies $\mathrm{C}$, et. al. Consensus review of optimal perioperative care in colorectal surgery. Enhanced Recovery After Surgery (ERAS) Group recommendations. Arch Surg 2009;144: 961-9.

\section{Macaskill 2001}

Macaskill P, Walter SD, Irwig L. A comparison of methods to detect publication bias in meta-analysis.. Statistics in Medicine 2001;20:641-54.

\section{Moher 1998}

Moher D, Pham B, Jones A, Cook DJ, Jadad AR, Moher $M$, et al.Does quality of reports of randomised trials affect estimates of intervention efficacy reported in meta-analyses? . Lancet 1998;352(9128):609-13.

Sammour 2010

Sammour T, Zargar-Shoshtari K, Bhat A, Kahokehr A, Hill AG. A programme of Enhanced Recovery After Surgery (ERAS) is a cost-effective intervention in elective colonic surgery.. $N Z$ Med J 2010 Jul 30;123(1319):61-70.

\section{Schultz 1995}

Schulz KF, Chalmers I, Hayer R, Altman D. Empirical evidence of bias.. JAMA 1995;273(5):408-12.

\section{Spanjersberg 2009}

Spanjersberg, W.R, Reurings, J, Laarhoven, van, C.H.J.M. Fast track surgery versus conventional recovery strategies 
for colorectal surgery.. Cochrane Database of Systematic Reviews 2009, Issue 2009 issue 1. [DOI: 10.1002/ 14651858.CD007635.]

\section{Tjandra 2006}

Tjandra JJ, Chan MK. Systematic review on the shortterm outcome of laparoscopic resection for colon and rectosigmoid cancer.. Colorectal Dis 2006 Jun;8(5):375-88.

\section{Varadhan 2010}

Krishna K Varadhan, Keith R. Neal, Cornelius H.C. DeJong, et al.The enhanced recovery after surgery (ERAS) pathway for patients undergoing major elective open colorectal surgery: A meta-analysis of randomized controlled trials.. Clinical Nutrition 2010;doi:10.1016/ j.clnu.2010.01.004:1-7.

\section{Walter 2009}

Walter, C. J.Collin, J.Dumville, J. C.Drew, P. J.Monson, J. R. Enhanced recovery in colorectal resections: A systematic review and meta-analysis. Colorectal Disease 2009;11(4): 344-353.

\section{Weeks 2002}

Weeks JC, Nelson H, Gelber S et al for the Clinical Outcomes of Surgical Therapy (COST) study group. Short-term quality-of-life outcomes following laparoscopicassisted colectomy vs open colectomy for colon cancer: a randomized trial. JAMA 2002;287:321-8.

\section{Weitz 2005}

J. Weitz, M. Koch, J. Debus, T. Hohler, P. R. Galle, M. W. Buchlers. Colorectal cancer. Lancet 2005 Jan 8-14;365( (9454)):153-65.

\section{Wilmore 2001}

Wilmore, D. W.Kehlet, H. Management of patients in fast track surgery. BMJ February 24, 2001;322(7284):473-6.

\section{Wind 2006}

Wind, J.Polle, S. W.Fung Kon Jin, P. H.Dejong, C. H.von Meyenfeldt, M. F.Ubbink, D. T.Gouma, D. J.Bemelman, W. A. Systematic review of enhanced recovery programmes in colonic surgery. Br J Surg July 2006;93(7):800-9.

* Indicates the major publication for the study 


\section{CHARACTERISTICS OF STUDIES}

\section{Characteristics of included studies [ordered by study ID]}

\section{Anderson 2003}

\begin{tabular}{|c|c|c|}
\hline Methods & \multicolumn{2}{|c|}{$\begin{array}{l}\text { Mono center RCT } \\
\text { - Loss to follow-up: } 0 \\
\text { - Intention to treat: ns } \\
\text { - Sample size calculations: no } \\
\text { - Contemporary groups: yes } \\
\text { - Exclusion after randomisation: } 2 \text { (peri-operative change in resection) }\end{array}$} \\
\hline Participants & \multicolumn{2}{|c|}{$\begin{array}{l}\text { Diagnosis: } 11 \text { intervention } / 7 \text { control malignant disease } \\
\text { inclusion: patients living independently at home needing elective hemicolectomy left/right } \\
\text { exclusion criteria: } \mathrm{nr}\end{array}$} \\
\hline Interventions & \multicolumn{2}{|c|}{$\begin{array}{l}\text { traditional surgical care vs ERAS protocol } \\
\text { no predefined ERAS interventions: } 12 \text { ERAS/ } 0 \text { conventional } \\
\text { Location: right hemicolectomy } 9 / 5 \text {, left hemicolectomy } 5 / 6\end{array}$} \\
\hline Outcomes & \multicolumn{2}{|c|}{$\begin{array}{l}\text { follow up: } 30 \text { days } \\
\text { Primary and secondary outcomes: length of hospital stay. } \\
\text { Measured outcomes: Hand grip strength, FEV1, time to return to normal diet, pain and fatigue } \\
\text { scores }\end{array}$} \\
\hline \multicolumn{3}{|l|}{ Notes } \\
\hline \multicolumn{3}{|l|}{ Risk of bias } \\
\hline Bias & Authors' judgement & Support for judgement \\
\hline Adequate sequence generation? & Unclear risk & Envelope randomisation, sequence unclear. \\
\hline Allocation concealment? & High risk & $\begin{array}{l}\text { All patients cared for by single surgeon; known } \\
\text { treatment protocol }\end{array}$ \\
\hline $\begin{array}{l}\text { Blinding? } \\
\text { All outcomes }\end{array}$ & High risk & $\begin{array}{l}\text { nor patient nor surgeon blinded, outcome asses- } \\
\text { sor unclear. }\end{array}$ \\
\hline Free of selective reporting? & Low risk & all data reported \\
\hline Free of other bias? & High risk & $\begin{array}{l}\text { both patient groups cared for on same ward, pos- } \\
\text { sibly introducing confounding in treatment ef- } \\
\text { fect }\end{array}$ \\
\hline
\end{tabular}


Delaney 2003

\begin{tabular}{|c|c|c|}
\hline Methods & \multicolumn{2}{|c|}{$\begin{array}{l}\text { Mono center RCT } \\
\text { - Loss to follow-up: } 0 \\
\text { - Intention to treat: Yes } \\
\text { - Sample size calculations: Yes, based on own data } 64 \text { patients needed } \\
\text { - Contemporary groups: yes, ERAS patients were significantly older } \\
\text { - Exclusion after randomisation: } 0\end{array}$} \\
\hline Participants & \multicolumn{2}{|c|}{$\begin{array}{l}\text { Diagnosis: Intervention Crohn } 9 / \mathrm{UC} 8 / \text { malignant } 9 / \text { diverse } 3 / \text { other } 2 \text { Control } 9 / 17 / 2 / 4 / 1 \\
\text { inclusion: All patient with elective segmental intestinal or rectal resection by laparotomy, including } \\
\text { re operations, pelvic surgery and comorbidity } \\
\text { exclusion: loop ileostomy closure and ventral hernia repair without scheduled intestinal resection }\end{array}$} \\
\hline Interventions & \multicolumn{2}{|c|}{$\begin{array}{l}\text { traditional surgical care vs ERAS protocol } \\
\text { no predefined ERAS interventions: } 4 \text { ERAS/ } 0 \text { conventional } \\
\text { Location: small intestine } 5 / 5 \text {, colon } 6 / 11 \text {, small intestine+rectum } 6 / 15 \text {, colon+rectum } 7 / 7\end{array}$} \\
\hline Outcomes & \multicolumn{2}{|c|}{$\begin{array}{l}\text { follow up: } 30 \text { days } \\
\text { Primary outcome: primary and total length of hospital stay. } \\
\text { Secondaryoutcome: effect of patients }<70 \text {, effect of surgeon experience, effect of diverting ileostomy } \\
\text { Measured outcomes: LOS, TLOS, enteric function, reinsertion nasogastric tubes, readmissions, } \\
\text { complications, Pain scores, quality of life scores, hospital satisfaction }\end{array}$} \\
\hline Notes & \multicolumn{2}{|c|}{$\begin{array}{l}\text { patients under } 70 \text { more effect, ERAS patients cared for by ERAS surgeon more effect (ns), no effect } \\
\text { of ileostomy }\end{array}$} \\
\hline \multicolumn{3}{|l|}{ Risk of bias } \\
\hline Bias & Authors' judgement & Support for judgement \\
\hline Adequate sequence generation? & Unclear risk & $\begin{array}{l}\text { sealed envelopes, created by biostatistics depart- } \\
\text { ment. envelope selection method unspecified }\end{array}$ \\
\hline Allocation concealment? & High risk & \\
\hline $\begin{array}{l}\text { Blinding? } \\
\text { All outcomes }\end{array}$ & High risk & nor patients nor surgeons \\
\hline Free of selective reporting? & Low risk & all recorded data reported \\
\hline Free of other bias? & High risk & $\begin{array}{l}\text { both patient groups cared for on same ward, pos- } \\
\text { sibly introducing confounding in treatment ef- } \\
\text { fect }\end{array}$ \\
\hline
\end{tabular}

Fast track surgery versus conventional recovery strategies for colorectal surgery (Review) 


\begin{tabular}{|c|c|}
\hline Methods & $\begin{array}{l}\text { Mono centre RCT } \\
\text { - Loss to follow-up: } 0 \\
\text { - Intention to treat:no } \\
\text { - Sample size calculations: yes, } 19 / \text { group } \\
\text { - Contemporary groups: yes } \\
\text { - Exclusion after randomisation: } 5 \text { ( } 2 \text { emergency surgery, } 2 \text { envisaged to require extensive hospital } \\
\text { stay unrelated to surgery, } 1 \text { no resection) }\end{array}$ \\
\hline Participants & $\begin{array}{l}\text { Diagnosis: malignant ERAS12/ conventional } 15 \\
\text { inclusion: All patients requiring elective colorectal surgery, living independently at home } \\
\text { exclusion: Age }<18 \text {, pregnancy, intolerance to pro-/prebiotic's, contraindication to one or more } \\
\text { optimisation strategy, contraindications to early discharge, prescribed medications that may inde- } \\
\text { pendently prolong hospital stay, advanced malignancy, palliative or emergency surgery, failure to } \\
\text { perform colonic or rectal resection }\end{array}$ \\
\hline Interventions & $\begin{array}{l}\text { traditional surgical care vs ERAS protocol } \\
\text { no predefined ERAS interventions: } 12 \text { ERAS/ } 1 \text { conventional, all analgesia same as optimised group } \\
\text { Location: (ERAS/conv) right hemicolectomy } 5 / 5 \text {, left hemicolectomy } 2 / 0 \text {, sigmoid } 0 / 2 \text {, Hartmann } \\
1 / 0 \text {, anterior } 5 / 10 \text {, subtotal colectomy } 3 / 0 \text {, pan proctocolectomy } 2 / 1 \text {, pan proctocolectomy with } \\
\text { pouch } 0 / 1 \text {, abdoperineal resection } 2 / 0\end{array}$ \\
\hline Outcomes & $\begin{array}{l}\text { follow up: } 30 \text { days } \\
\text { Primary outcome: hand grip strength } \\
\text { Secondary outcome: length of hospital stay } \\
\text { Measured outcomes: physiological function (spirometry, duration of catheterization, fluid balance, } \\
\text { time to mobilisation), Psychological function (cognitive, fatigue and pain scoring, analgesic re- } \\
\text { quirements), Gut function (duration of iv fluids and time to tolerance of fluids and diet), Clinical } \\
\text { outcome (length of hospital stay, complications and death, need for readmissions, general practi- } \\
\text { tioner visits) }\end{array}$ \\
\hline
\end{tabular}

Notes

Risk of bias

\begin{tabular}{l|l|l}
\hline Bias & Authors judgement & Support for judgement \\
\hline Adequate sequence generation? & Unclear risk & $\begin{array}{l}\text { Randomization using sealed envelopes, sequence } \\
\text { unknown }\end{array}$ \\
\hline $\begin{array}{l}\text { Allocation concealment? } \\
\text { Blinding? }\end{array}$ & High risk & $\begin{array}{l}\text { in an attempt to decrease bias, discharge criteria } \\
\text { were standardized }\end{array}$ \\
\hline $\begin{array}{l}\text { All outcomes } \\
\text { Free of selective reporting? }\end{array}$ & Low risk risk & nor patient, nor surgeon, nor outcome assessor \\
\hline Free of other bias? & High risk & all outcome measures registered also reported \\
\hline
\end{tabular}


Khoo 2007

\begin{tabular}{|c|c|c|}
\hline Methods & \multicolumn{2}{|c|}{$\begin{array}{l}\text { Mono centre RCT } \\
\text { - Loss to follow-up: unclear } \\
\text { - Intention to treat: yes, except for pre-/perioperative exclusions } \\
\text { - Sample size calculations: yes, } 45 / \text { group. } \\
\text { - Contemporary groups: yes, also pre-randomisation stratified for sex and planned TME } \\
\text { - Exclusion after randomisation: } 11 \text { (7 post randomisation metastatic disease, } 1 \text { not operated, } 3 \\
\text { withdrew consent before surgery, } 1 \text { after surgery; data included in intention to treat analysis) }\end{array}$} \\
\hline Participants & \multicolumn{2}{|c|}{$\begin{array}{l}\text { Diagnosis: Colonic surgery (ERAS/ conventional) } 22 / 25 \text {, rectal surgery with TME } 13 / 10 \\
\text { inclusion: All elective colorectal malignancy, able to walk }>100 \mathrm{~m} \text {, curative surgery } \\
\text { exclusion: palliative surgery, depression, contraindication for epidural analgesia }\end{array}$} \\
\hline Interventions & \multicolumn{2}{|c|}{$\begin{array}{l}\text { traditional surgical care vs ERAS protocol } \\
\text { no predefined ERAS interventions: } 9 \text { ERAS ( } 10 \text {, because partial transverse incisions performed)/ } 2 \\
\text { conventional }(3 \text {, also partial transverse incisions) } \\
\text { Location: colon } 22 / 25 \text {, rectal } 13 / 10\end{array}$} \\
\hline Outcomes & \multicolumn{2}{|c|}{$\begin{array}{l}\text { follow up: 10-14 days } \\
\text { Primary outcome: length of hospital stay } \\
\text { Secondaryoutcome: complications, mortality, readmissions } \\
\text { Measured outcomes: intravenous volume given first } 47 \text { hours, achievement of discharge criteria, } \\
\text { readmissions, complications, postdischarge outcomes, length of hospital stay, }\end{array}$} \\
\hline \multicolumn{3}{|l|}{ Notes } \\
\hline \multicolumn{3}{|l|}{ Risk of bias } \\
\hline Bias & Authors' judgement & Support for judgement \\
\hline Adequate sequence generation? & Low risk & $\begin{array}{l}\text { Central randomisation using random number } \\
\text { generator, randomised by telephone }\end{array}$ \\
\hline Allocation concealment? & High risk & patients and staff aware of allocation \\
\hline $\begin{array}{l}\text { Blinding? } \\
\text { All outcomes }\end{array}$ & High risk & $\begin{array}{l}\text { nor patients, nor staff, patients treated in separate } \\
\text { hospital wards }\end{array}$ \\
\hline Free of selective reporting? & Unclear risk & no reporting on measured outcome \\
\hline Free of other bias? & Low risk & \\
\hline
\end{tabular}




\begin{tabular}{|c|c|c|}
\hline Methods & \multicolumn{2}{|c|}{$\begin{array}{l}\text { Multi-center RCT ( } 4 \text { hospitals) } \\
\text { - Loss to follow-up: } 0 \\
\text { - Intention to treat: yes } \\
\text { - Sample size calculations: yes, } 231 \text { patients per group; After interim analysis, study stopped at } 156 \\
\text { inclusions, } 151 \text { patients completed study } \\
\text { - Contemporary groups: slight difference in median age, indications not listed } \\
\text { - Exclusion after randomisation: } 0\end{array}$} \\
\hline Participants & \multicolumn{2}{|c|}{$\begin{array}{l}\text { Diagnosis: not listed } \\
\text { inclusion: older than } 18 \text {, open elective colonic resection with primary anastomosis } \\
\text { exclusion: emergency surgery, contraindications to epidural analgesia, scheduled total colectomy } \\
\text { or rectum resection and preoperatively immobile patients }\end{array}$} \\
\hline Interventions & \multicolumn{2}{|c|}{$\begin{array}{l}\text { traditional surgical care vs ERAS protocol } \\
\text { no predefined ERAS interventions: } 6 \text { ERAS/ } 4 \text { conventional } \\
\text { Location: Left hemicolectomy or sigmoidectomy } 50 / 51 \text {, transverse colectomy } 0 / 2 \text {, right hemicolec- } \\
\text { tomy } 26 / 22\end{array}$} \\
\hline Outcomes & \multicolumn{2}{|c|}{$\begin{array}{l}\text { follow up: } 30 \text { days } \\
\text { Primary outcome: complications } \\
\text { Secondaryoutcome: } \\
\text { Measured outcomes: total and intra-operative fluid administration, oral food intake and mobilisa- } \\
\text { tion time }\end{array}$} \\
\hline Notes & \multicolumn{2}{|c|}{ oral food intake and mobilisation time were registered by patient self-assessment } \\
\hline \multicolumn{3}{|l|}{ Risk of bias } \\
\hline Bias & Authors' judgement & Support for judgement \\
\hline Adequate sequence generation? & Low risk & online software, stratified for centre \\
\hline Allocation concealment? & High risk & \\
\hline $\begin{array}{l}\text { Blinding? } \\
\text { All outcomes }\end{array}$ & High risk & \\
\hline Free of selective reporting? & Low risk & \\
\hline Free of other bias? & High risk & $\begin{array}{l}\text { certain outcome measures recorded by patient } \\
\text { self assessment; since ERAS patients are encour- } \\
\text { aged to eat and mobilise, reporting bias may be } \\
\text { present. Both patient groups cared for on same } \\
\text { ward, possibly introducing confounding in treat- } \\
\text { ment effect }\end{array}$ \\
\hline
\end{tabular}


Serclova 2009

\begin{tabular}{|c|c|}
\hline Methods & $\begin{array}{l}\text { Mono centre RCT } \\
\text { - Loss to follow-up: } 0 \\
\text { - Intention to treat: yes } \\
\text { - Sample size calculations: Yes, depending on outcome between } 30 \text { and } 47 \text { patients per group } \\
\text { - Contemporary groups: Yes, although sex significantly different between intervention and control } \\
\text { - Exclusion after randomisation: Yes, } 2 \text { in ERAS group }\end{array}$ \\
\hline Participants & $\begin{array}{l}\text { Diagnosis: Intervention Crohn 42, UC } 4 \text { FAP } 1 \text {, Carcinoma 3, other } 1 \text {. Control 38/5/4/4/1 } \\
\text { inclusion: All patients ASA I or II, aged between } 18-70 \text {, with elective open intestinal resection } \\
\text { exclusion: age }<19,>70 \text {, ASA III/IV, Pregnancy, Pelvic radiation and multiorgan resection }\end{array}$ \\
\hline Interventions & $\begin{array}{l}\text { traditional surgical care vs ERAS protocol } \\
\text { no predefined ERAS interventions: } 11 \text { ERAS/ } 1 \text { conventional ( } 68 \% \text { epidural) } \\
\text { Location:unspecified }\end{array}$ \\
\hline Outcomes & $\begin{array}{l}\text { follow up: } 30 \text { days } \\
\text { Primary outcome: safety of protocol } \\
\text { Secondaryoutcome: improved pain management, shortened length of stay } \\
\text { Measured outcomes: length of stay, VAS pain scores, time to bowel movement, time to normal diet, } \\
\text { complications }\end{array}$ \\
\hline
\end{tabular}

\section{Risk of bias}

\begin{tabular}{|c|c|c|}
\hline Bias & Authors' judgement & Support for judgement \\
\hline Adequate sequence generation? & Low risk & sequence generated by statistician \\
\hline Allocation concealment? & High risk & nor patients nor staff \\
\hline $\begin{array}{l}\text { Blinding? } \\
\text { All outcomes }\end{array}$ & High risk & nor patients, nor staff or outcome assessor \\
\hline Free of selective reporting? & High risk & $\begin{array}{l}\text { BMI and severity of surgery recorded, not re- } \\
\text { ported }\end{array}$ \\
\hline Free of other bias? & High risk & $\begin{array}{l}\text { possible selection bias, since centre is specialized } \\
\text { for IBD surgery. Both patient groups cared for on } \\
\text { same ward, possibly introducing confounding in } \\
\text { treatment effect }\end{array}$ \\
\hline
\end{tabular}

$\mathrm{UC}=$ Ulcerative colitis, $\mathrm{nr}=$ not reported, $\mathrm{ns}=$ not significant, $\mathrm{LOS}=$ primary length of stay, TLOS= total length of stay, FAP=Familiar adenomatosis polyposis 
Characteristics of excluded studies [ordered by study ID]

\begin{tabular}{|c|c|}
\hline Study & Reason for exclusion \\
\hline Basse 2005 & All participants received enhanced recovery programs, randomisation was between open and laparoscopic procedure \\
\hline Holte 2007 & Both groups receiving ERAS treatment \\
\hline King 2006 & both groups received ERAS treatment \\
\hline King 2008 & both groups received ERAS treatment \\
\hline Kuzma 2008 & only patients with appendectomy included \\
\hline Liu 2008 & poor quality, pseudo randomised \\
\hline MacKay 2006 & both groups received ERAS treatment \\
\hline Noblett 2006 & all patients received ERAS pathway \\
\hline Raue 2004 & $\begin{array}{l}\text { comparison laparoscopic ERAS vs conventional; insufficient randomisation, conventional pathway contains multiple } \\
\text { ERAS interventions }\end{array}$ \\
\hline Xu 2007 & pseudo randomised trial \\
\hline Zutshi 2004 & Outcome values restricted to activity and length of stay, no follow up \\
\hline Zutshi 2005 & All patients received ERAS pathways, and randomised between epidural analgesia and PCA morphine \\
\hline
\end{tabular}

\section{Characteristics of ongoing studies [ordered by study [D]}

\section{Reurings 2009}

\begin{tabular}{l|l}
\hline Trial name or title & $\begin{array}{l}\text { A prospective cohort study to investigate cost-minimisation, of Traditional open, open fAst track recovery } \\
\text { and laParoscopic fASt track multimodal management, for surgical patients with colon carcinomas. (TAPAS } \\
\text { study) }\end{array}$ \\
\hline Methods & Prospective cohort trial, multicenter. 3 cohorts, included sequentially \\
\hline Participants & Patients, ASA 1 or 2 aged 18 or over with colon carcinoma without metastasis \\
\hline Interventions & three cohorts; conventional treatment, ERAS treatment, laparoscopic surgery with ERAS treatment \\
\hline Outcomes & Morbidity and mortality, cost effectiveness, quality of life \\
\hline Starting date & $1-1-07$
\end{tabular}

Fast track surgery versus conventional recovery strategies for colorectal surgery (Review) 
Reurings 2009 (Continued)

Contact information J.C Reurings, St Elisabeth hospital Tilburg, department of surgery

Notes

Wind 2006

Trial name or title Perioperative strategy in colonic surgery; LAparoscopy and/or FAst track multimodal management versus standard care (LAFA trial)

\begin{tabular}{ll}
\hline Methods & Randomized Clinical Trial; double blinded, multicenter trial with a 2 × 2 balanced factorial design \\
\hline Participants & $\begin{array}{l}\text { Patients eligible for segmental colectomy for malignant colorectal disease i.e. right and left colectomy and } \\
\text { anterior resection will be randomised to either open or laparoscopic colectomy, and to either standard care or } \\
\text { the fast track program }\end{array}$
\end{tabular}

Interventions $\quad$ This factorial design produces four treatment groups; open colectomy with standard care (a), open colectomy with fast track program (b), laparoscopic colectomy with standard care (c), and laparoscopic surgery with fast track program $(\mathrm{d})$

Outcomes $\quad$ Primary outcome parameter is postoperative hospital length of stay including readmission within 30 days. Secondary outcome parameters are quality of life two and four weeks after surgery, overall hospital costs, morbidity, patient satisfaction and readmission rate

Starting date

Contact information Department of Surgery, Academic Medical Center Amsterdam, The Netherlands. j.wind@amc.uva.nl

Notes Inclusion for this study has finished, data analysis is underway 
DATA AND ANALYSES

Comparison 1. Primary analyses ERAS versus conventional

\begin{tabular}{lcclc} 
Outcome or subgroup title & $\begin{array}{c}\text { No. of } \\
\text { studies }\end{array}$ & $\begin{array}{c}\text { No. of } \\
\text { participants }\end{array}$ & \multicolumn{1}{c}{ Statistical method } & Effect size \\
\hline 1 Mortality & 4 & 237 & Risk Ratio (M-H, Fixed, 95\% CI) & $0.53[0.12,2.38]$ \\
2 All complications & 4 & 237 & Risk Ratio (M-H, Random, 95\% CI) & $0.52[0.38,0.71]$ \\
3 Major Complications & 3 & 134 & Risk Ratio (M-H, Random, 95\% CI) & $0.45[0.09,2.32]$ \\
4 Minor complications & 3 & 134 & Risk Ratio (M-H, Random, 95\% CI) & $0.63[0.37,1.05]$ \\
5 Undefined complications & 1 & 103 & Risk Ratio (M-H, Random, 95\% CI) & $0.42[0.23,0.75]$ \\
6 Readmissions & 4 & 237 & Risk Ratio (M-H, Random, 95\% CI) & $0.87[0.08,9.39]$ \\
7 hospital stay & 4 & 237 & Mean Difference (IV, Fixed, 95\% CI) & $-2.94[-3.69,-2.19]$ \\
\hline
\end{tabular}

Comparison 2. Sensitivity analyses ERAS vs conventional

\begin{tabular}{lccll} 
Outcome or subgroup title & $\begin{array}{c}\text { No. of } \\
\text { studies }\end{array}$ & $\begin{array}{c}\text { No. of } \\
\text { participants }\end{array}$ & \multicolumn{1}{c}{ Statistical method } & Effect size \\
\hline 1 mortality & 6 & 452 & Risk Ratio (M-H, Random, 95\% CI) & $0.53[0.09,3.15]$ \\
2 Total complications & 6 & 452 & Risk Ratio (M-H, Fixed, 95\% CI) & $0.51[0.39,0.67]$ \\
3 Major complications & 5 & 349 & Risk Ratio (M-H, Fixed, 95\% CI) & $0.50[0.28,0.92]$ \\
4 Minor complications & 5 & 349 & Risk Ratio (M-H, Fixed, 95\% CI) & $0.57[0.38,0.85]$ \\
5 Undefined complications & 1 & 103 & Risk Ratio (M-H, Fixed, 95\% CI) & $0.42[0.23,0.75]$ \\
6 readmissions & 6 & 452 & Risk Ratio (M-H, Fixed, 95\% CI) & $0.79[0.36,1.76]$ \\
7 hospital stay & 6 & 452 & Mean Difference (IV, Random, 95\% CI) & $-2.51[-3.54,-1.47]$ \\
\hline
\end{tabular}




\section{Analysis I.I. Comparison I Primary analyses ERAS versus conventional, Outcome I Mortality.}

Review: Fast track surgery versus conventional recovery strategies for colorectal surgery

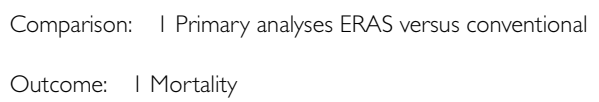

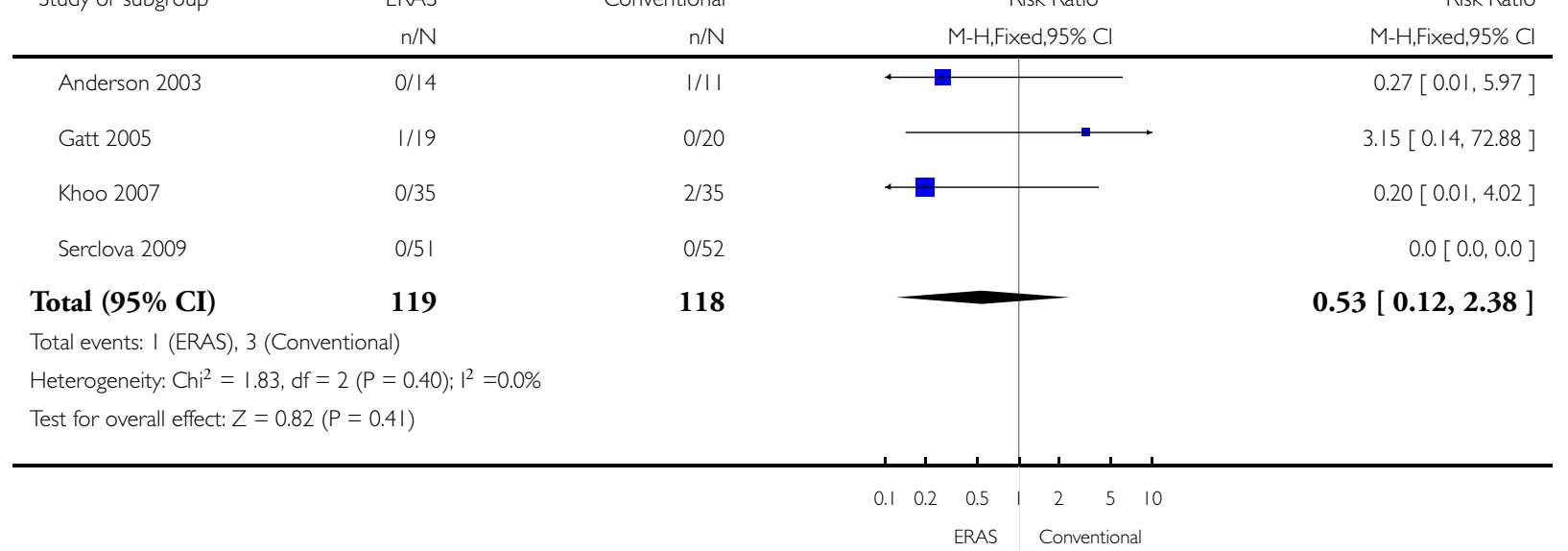




\section{Analysis I.2. Comparison I Primary analyses ERAS versus conventional, Outcome 2 All complications.}

Review: Fast track surgery versus conventional recovery strategies for colorectal surgery

Comparison: I Primary analyses ERAS versus conventional

Outcome: 2 All complications

Study or subgroup

ERAS

Conventiona

Risk Ratio

M-

$\mathrm{H}$, Random,95\%

\begin{tabular}{lll} 
& $\mathrm{n} / \mathrm{N}$ & $\mathrm{n} / \mathrm{N}$ \\
\hline Anderson 2003 & $5 / 14$ & $7 / 11$
\end{tabular}

Cl

Risk Ratio

$H$,Random $95 \%$

$\longrightarrow$

Gatt 2005

$9 / 19$

15/20

Khoo 2007

$9 / 35$

18/35

Serclova 2009

||$/ 5 \mid$

27/52

119

118
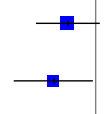

$\longrightarrow$

Total (95\% CI)

Total events: 34 (ERAS), 67 (Conventional)

Heterogeneity: $\mathrm{Tau}^{2}=0.0 ; \mathrm{Chi}^{2}=1.17, \mathrm{df}=3(\mathrm{P}=0.76) ;\left.\right|^{2}=0.0 \%$

Test for overall effect: $Z=4.07(P=0.000046)$

Test for subgroup differences: Not applicable $\begin{array}{lllllll}0.1 & 0.2 & 0.5 & 1 & 2 & 5 & 10\end{array}$

Favours eras Favours conventional 


\section{Analysis I.3. Comparison I Primary analyses ERAS versus conventional, Outcome 3 Major Complications.}

Review: Fast track surgery versus conventional recovery strategies for colorectal surgery

Comparison: I Primary analyses ERAS versus conventional

Outcome: 3 Major Complications

$n / N \quad n / N$

Anderson 2003

$1 / 14$

$2 / 11$

Gatt 2005

$4 / 19$

$3 / 20$

Khoo 2007

$1 / 35$

9/35

68

66

Total (95\% CI)

Total events: 6 (ERAS), 14 (Conventional)

Heterogeneity: $\operatorname{Tau}^{2}=1.20 ; \mathrm{Chi}^{2}=4.70, \mathrm{df}=2(\mathrm{P}=0.10) ; \mathrm{I}^{2}=57 \%$

Test for overall effect: $Z=0.95(P=0.34)$

Test for subgroup differences: Not applicable

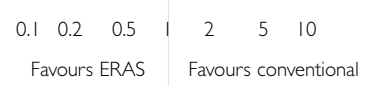

Fast track surgery versus conventional recovery strategies for colorectal surgery (Review) 


\section{Analysis I.4. Comparison I Primary analyses ERAS versus conventional, Outcome 4 Minor complications.}

\begin{tabular}{|c|c|c|c|c|c|}
\hline \multicolumn{6}{|c|}{ Comparison: I Primary analyses ERAS versus conventional } \\
\hline \multicolumn{6}{|c|}{ Outcome: 4 Minor complications } \\
\hline Study or subgroup & $\begin{array}{r}\text { ERAS } \\
\mathrm{n} / \mathrm{N} \\
\end{array}$ & Conventional & $\begin{array}{c}\text { Risk Ratio } \\
\text { M- } \\
\text { H,Random,95\% } \\
\text { Cl }\end{array}$ & Weight & $\begin{array}{c}\text { Risk Ratio } \\
\text { M- } \\
\text { H,Random,95\% } \\
\text { Cl }\end{array}$ \\
\hline Anderson 2003 & $4 / 14$ & $5 / 11$ & 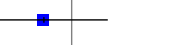 & $23.8 \%$ & $0.63[0.22,1.80]$ \\
\hline Gatt 2005 & $5 / 19$ & $12 / 20$ & 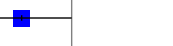 & $37.9 \%$ & $0.44[0.19,1.01]$ \\
\hline Khoo 2007 & $8 / 35$ & $9 / 35$ & + & $38.3 \%$ & $0.89[0.39,2.04]$ \\
\hline Total $(95 \% \mathrm{CI})$ & 68 & 66 & $\longrightarrow$ & $100.0 \%$ & $0.63[0.37,1.05]$ \\
\hline \multicolumn{6}{|c|}{ Total events: 17 (ERAS), 26 (Conventional) } \\
\hline \multicolumn{6}{|c|}{ Heterogeneity: $\operatorname{Tau}^{2}=0.0 ; \mathrm{Chi}^{2}=1.39, \mathrm{df}=2(\mathrm{P}=0.50) ; \mathrm{I}^{2}=0.0 \%$} \\
\hline \multicolumn{6}{|c|}{ Test for overall effect: $Z=1.79(P=0.074)$} \\
\hline \multicolumn{6}{|c|}{ Test for subgroup differences: Not applicable } \\
\hline
\end{tabular}

\section{Analysis I.5. Comparison I Primary analyses ERAS versus conventional, Outcome 5 Undefined complications.}

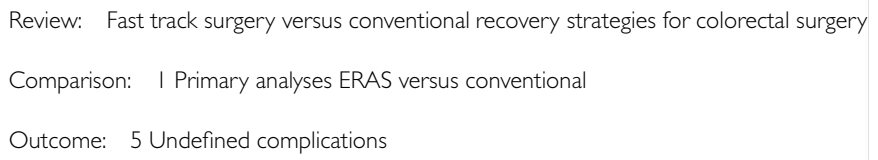

世-

Total (95\% CI)

51

Total events: I I (ERAS), 27 (Conventional)

Heterogeneity: not applicable

Test for overall effect: $Z=2.94(P=0.0033)$

Test for subgroup differences: Not applicable 


\section{Analysis I.6. Comparison I Primary analyses ERAS versus conventional, Outcome 6 Readmissions.}

Review: Fast track surgery versus conventional recovery strategies for colorectal surgery

Comparison: I Primary analyses ERAS versus conventional

Outcome: 6 Readmissions

Study or subgroup $\quad$ ERAS Conventional Risk Ratio

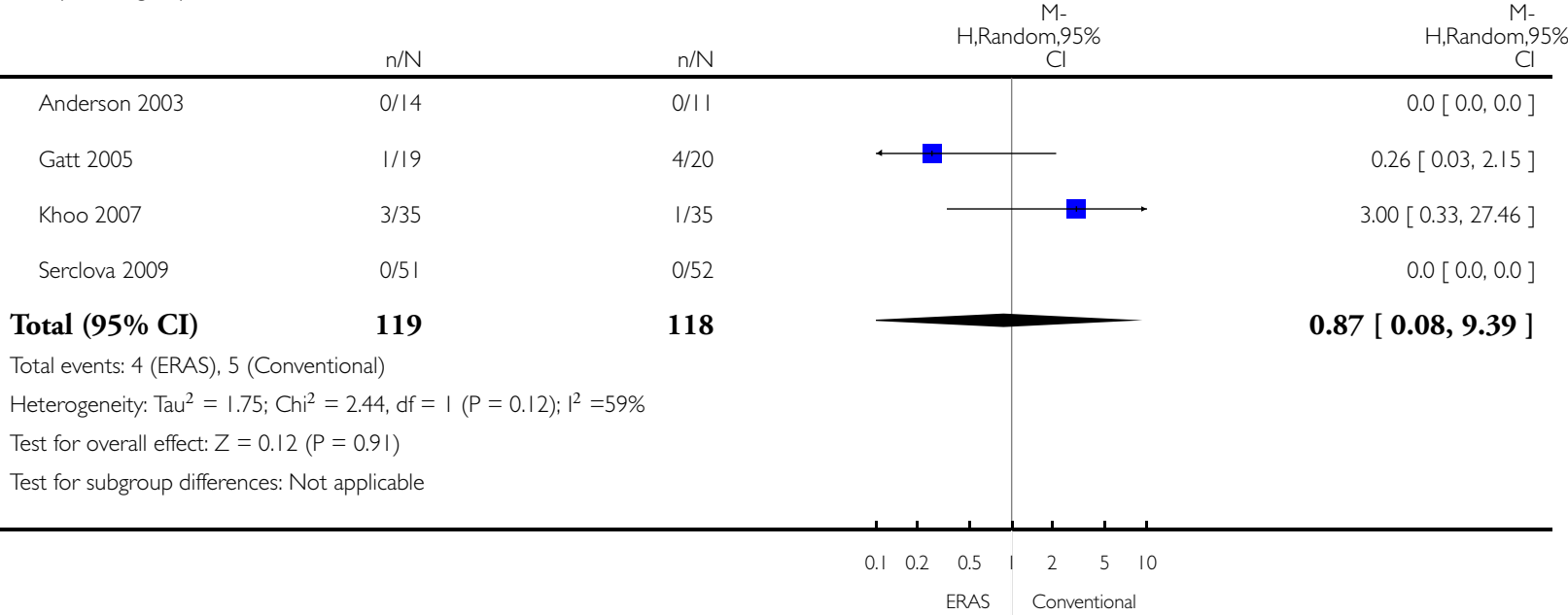




\section{Analysis I.7. Comparison I Primary analyses ERAS versus conventional, Outcome 7 hospital stay.}

Review: Fast track surgery versus conventional recovery strategies for colorectal surgery

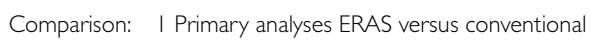

\begin{tabular}{|c|c|c|c|c|c|c|c|c|}
\hline \multirow{3}{*}{$\begin{array}{l}\text { Study or subgroup } \\
\text { Anderson } 2003\end{array}$} & \multirow{3}{*}{$\begin{array}{r}\text { ERAS } \\
N\end{array}$} & \multicolumn{2}{|r|}{ Conventional } & \multicolumn{3}{|c|}{$\begin{array}{r}\text { Mean } \\
\text { Difference }\end{array}$} & \multicolumn{2}{|r|}{$\begin{array}{r}\text { Mean } \\
\text { Difference }\end{array}$} \\
\hline & & Mean(SD)[days] & N & Mean(SD)[days] & \multicolumn{2}{|c|}{ IV,Fixed,95\% Cl } & & IV,Fixed,95\% Cl \\
\hline & & $4(1.8)$ & 11 & $7(2.1)$ & $\longleftrightarrow$ & & $23.4 \%$ & $-3.00[-4.56,-1.44]$ \\
\hline Gatt 2005 & 19 & $6.6(4.4)$ & 20 & $9(4.6)$ & & - & $7.1 \%$ & $-2.40[-5.22,0.42]$ \\
\hline Khoo 2007 & 35 & $5(8.5)$ & 35 & $7(14.75)$ & $\longleftarrow$ & & $1.8 \%$ & $-2.00[-7.64,3.64]$ \\
\hline Serclova 2009 & 51 & $7.4(1.3)$ & 52 & $10.4(3.1)$ & $\longrightarrow$ & & $67.8 \%$ & $-3.00[-3.92,-2.08]$ \\
\hline Total (95\% CI) & 119 & & 118 & & 4 & & $100.0 \%$ & $-2.94[-3.69,-2.19]$ \\
\hline \multicolumn{9}{|c|}{ Heterogeneity: $\mathrm{Chi}^{2}=0.27, \mathrm{df}=3(\mathrm{P}=0.97) ; \mathrm{I}^{2}=0.0 \%$} \\
\hline \multicolumn{9}{|c|}{ Test for overall effect: $Z=7.65(P<0.0000 I)$} \\
\hline \multicolumn{9}{|c|}{ Test for subgroup differences: Not applicable } \\
\hline & & & & & -2 & 2 & 4 & \\
\hline & & & & & ERAS & Conver & & \\
\hline
\end{tabular}




\section{Analysis 2.I. Comparison 2 Sensitivity analyses ERAS vs conventional, Outcome I mortality.}

Review: Fast track surgery versus conventional recovery strategies for colorectal surgery

Comparison: 2 Sensitivity analyses ERAS vs conventional

Outcome: I mortality

ERAS

conventional

Risk Ratio

M-

$n / N \quad n / N$

Anderson 2003

$0 / 14$

I/II

Delaney 2003

$0 / 31$

0/33

Gatt 2005

$1 / 19$

$0 / 20$

Khoo 2007

0/35

2/35

Muller 2009

0/76

0/75

Serclova 2009

Total (95\% CI)

al)

Total events: I (ERAS), 3 (conventional)

Heterogeneity: $\operatorname{Tau}^{2}=0.0 ; \mathrm{Chi}^{2}=1.83, \mathrm{df}=2(\mathrm{P}=0.40) ; \mathrm{I}^{2}=0.0 \%$

Test for overall effect: $Z=0.69(P=0.49)$

Test for subgroup differences: Not applicable

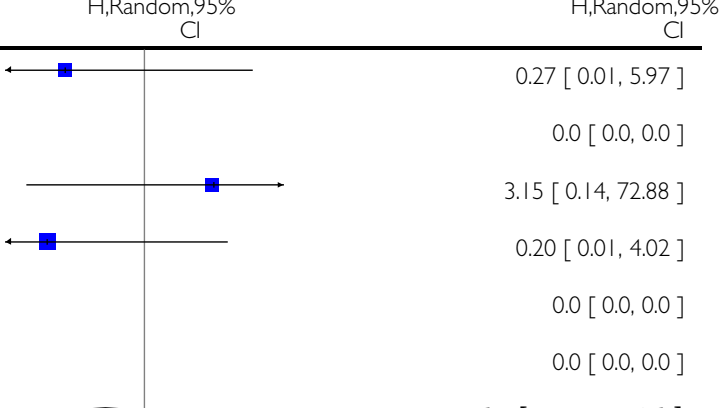

0.53 [ $0.09,3.15$ ] 


\section{Analysis 2.2. Comparison 2 Sensitivity analyses ERAS vs conventional, Outcome 2 Total complications.}

Review: Fast track surgery versus conventional recovery strategies for colorectal surgery

Comparison: 2 Sensitivity analyses ERAS vs conventional

Outcome: 2 Total complications

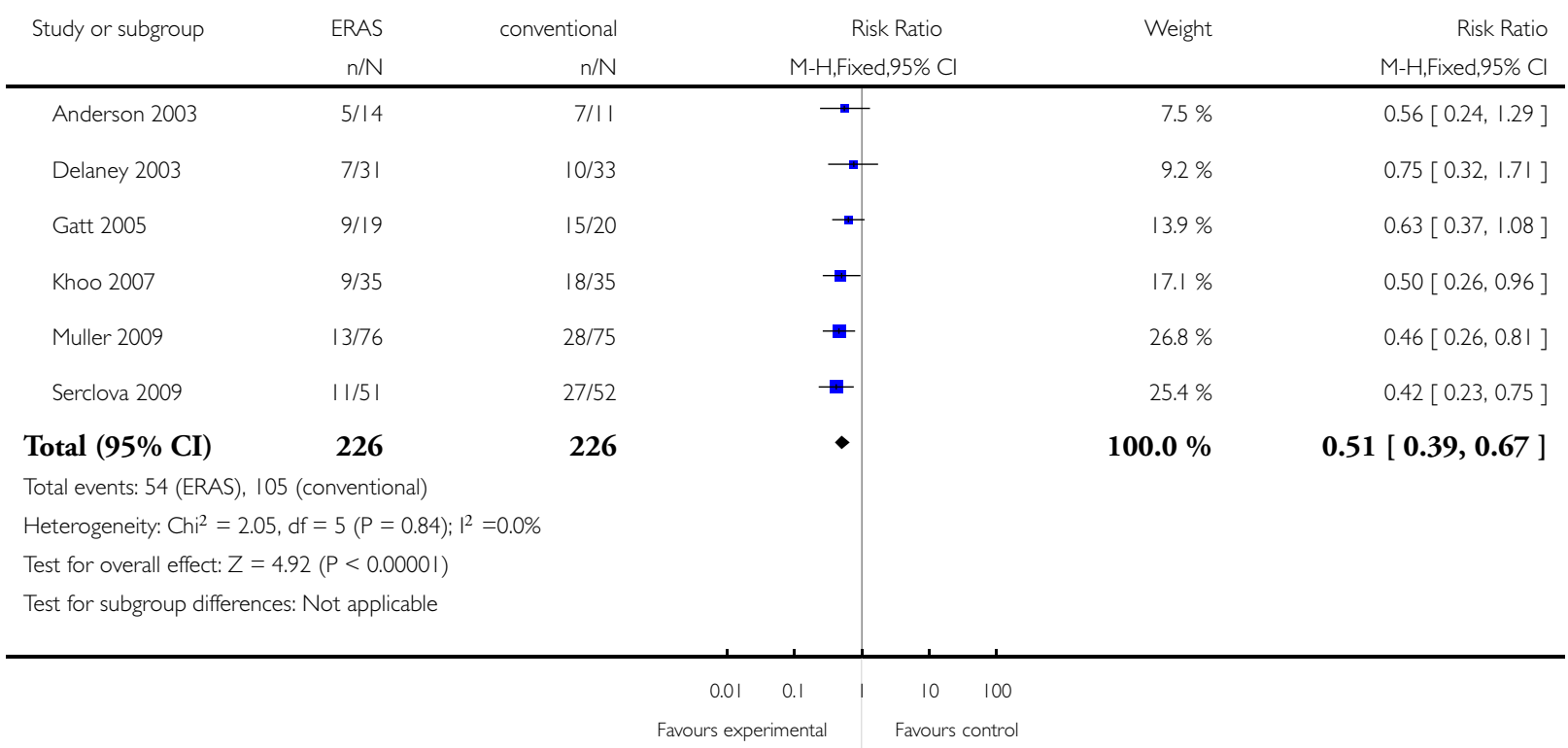


Analysis 2.3. Comparison 2 Sensitivity analyses ERAS vs conventional, Outcome 3 Major complications.

Review: Fast track surgery versus conventional recovery strategies for colorectal surgery

Comparison: 2 Sensitivity analyses ERAS vs conventional

Outcome: 3 Major complications

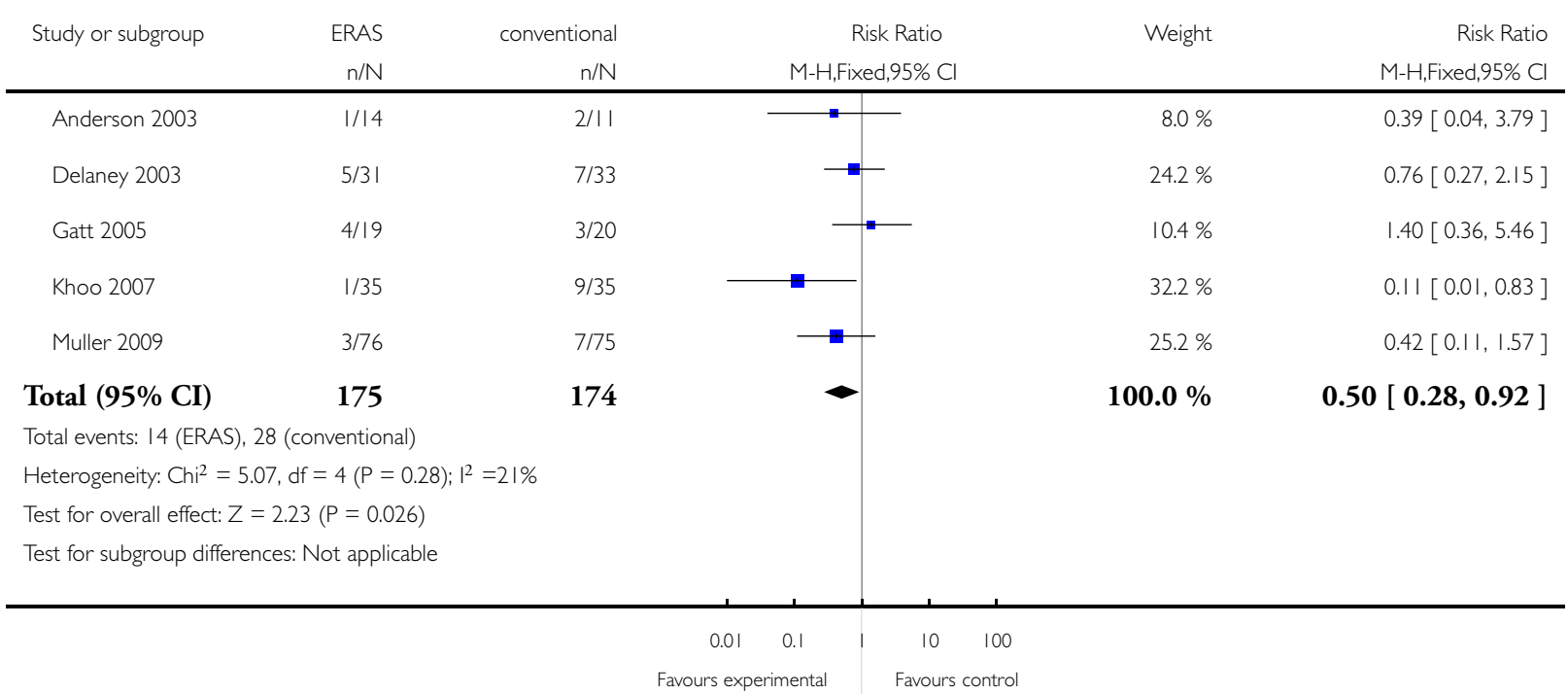


Analysis 2.4. Comparison 2 Sensitivity analyses ERAS vs conventional, Outcome 4 Minor complications.

Review: Fast track surgery versus conventional recovery strategies for colorectal surgery

Comparison: 2 Sensitivity analyses ERAS vs conventional

Outcome: 4 Minor complications

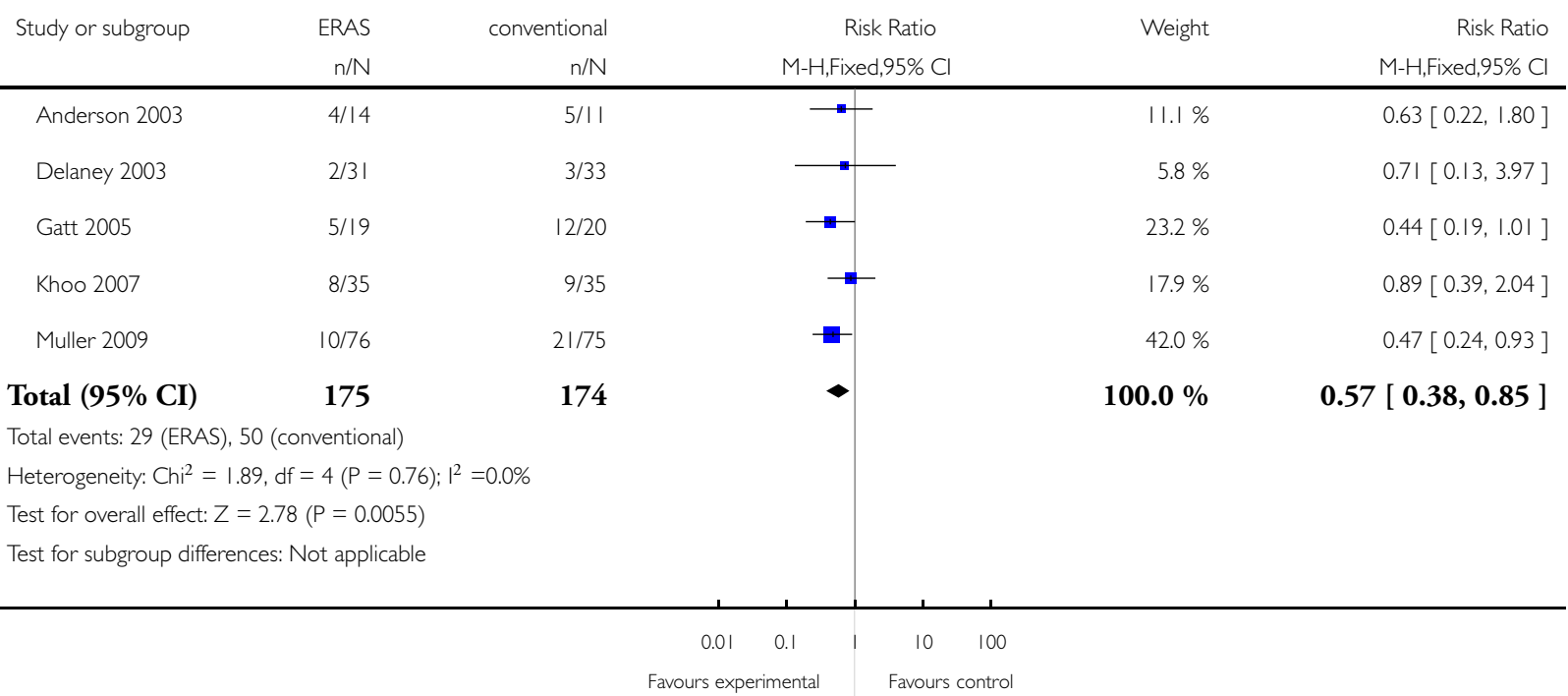

Analysis 2.5. Comparison 2 Sensitivity analyses ERAS vs conventional, Outcome 5 Undefined complications.

Review: Fast track surgery versus conventional recovery strategies for colorectal surgery

Comparison: 2 Sensitivity analyses ERAS vs conventional

Outcome: 5 Undefined complications

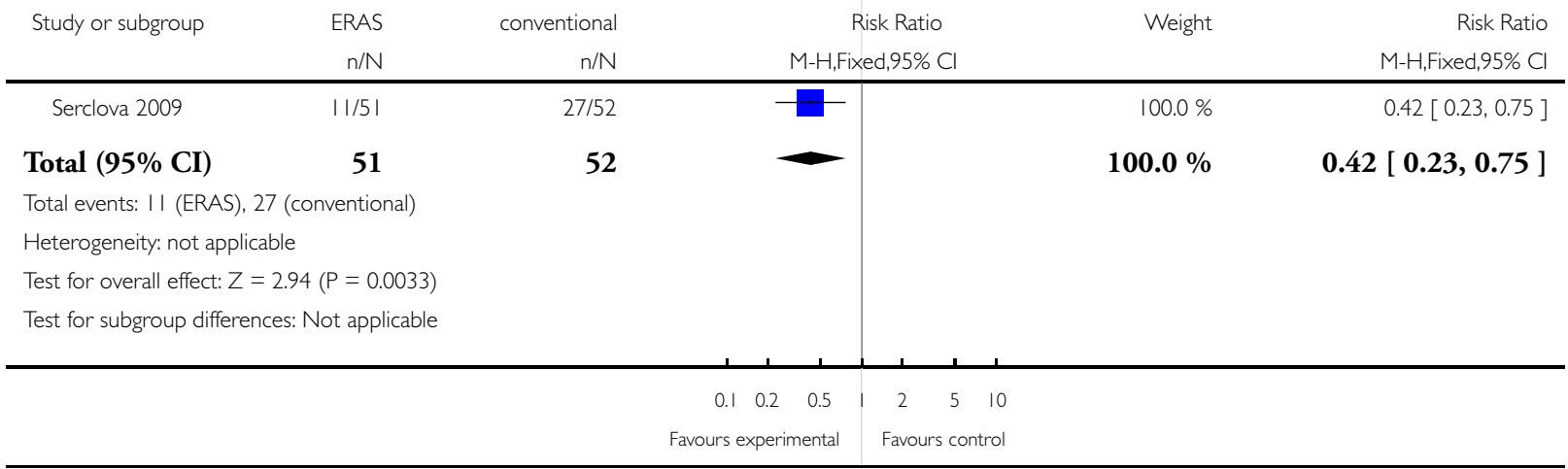

Fast track surgery versus conventional recovery strategies for colorectal surgery (Review)

Copyright @ 201 I The Cochrane Collaboration. Published by John Wiley \& Sons, Ltd. 


\section{Analysis 2.6. Comparison 2 Sensitivity analyses ERAS vs conventional, Outcome 6 readmissions.}

Review: Fast track surgery versus conventional recovery strategies for colorectal surgery

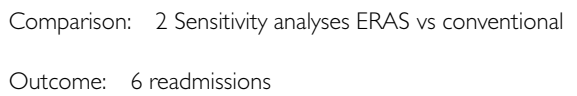

$\mathrm{n} / \mathrm{N}$

Anderson 2003

0/II

Delaney 2003

$3 / 31$

Risk Ratio M-H,Fixed,95\% Cl

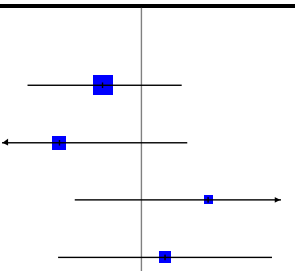

$$
\begin{array}{lllllll}
0.1 & 0.2 & 0.5 & 1 & 2 & 5 & 10 \\
& & \text { ERAS } & \multicolumn{3}{c}{\text { Conventional }}
\end{array}
$$

Risk Ratio M-H,Fixed,95\% Cl

$0.0[0.0,0.0]$

$0.53[0.15,1.95]$

$0.26[0.03,2.15]$

$3.00[0.33,27.46]$

$1.48[0.25,8.61]$

$0.0[0.0,0.0]$

$0.79[0.36,1.76]$ 


\section{Analysis 2.7. Comparison 2 Sensitivity analyses ERAS vs conventional, Outcome 7 hospital stay.}

Review: Fast track surgery versus conventional recovery strategies for colorectal surgery

Comparison: 2 Sensitivity analyses ERAS vs conventional

Outcome: 7 hospital stay

\begin{tabular}{|c|c|c|c|c|c|c|c|c|}
\hline \multirow{3}{*}{$\begin{array}{l}\text { Study or subgroup } \\
\text { Anderson } 2003\end{array}$} & \multirow{3}{*}{$\begin{array}{r}\text { ERAS } \\
\mathrm{N} \\
14\end{array}$} & \multirow{3}{*}{\multicolumn{2}{|c|}{$\frac{\begin{array}{r}\text { conventional } \\
N\end{array}}{\mathrm{II}}$}} & \multirow{3}{*}{$\frac{\text { Mean(SD)[days }]}{7(2.1)}$} & \multirow{2}{*}{\multicolumn{2}{|c|}{$\begin{array}{c}\text { Mean } \\
\text { Difference } \\
\text { IV,Random,95\% Cl }\end{array}$}} & \multirow{3}{*}{$\begin{array}{l}\text { Weight } \\
19.3 \%\end{array}$} & \multirow{3}{*}{$\begin{array}{r}\text { Mean } \\
\text { Difference } \\
\text { IV,Random,95\% Cl } \\
-3.00[-4.56,-1.44]\end{array}$} \\
\hline & & & & & & & & \\
\hline & & & & & \multirow[t]{2}{*}{$\hookrightarrow$} & & & \\
\hline Delaney 2003 & 31 & $5.2(2.5)$ & 33 & $5.8(3)$ & & & $21.7 \%$ & $-0.60[-1.95,0.75]$ \\
\hline Gatt 2005 & 19 & $6.6(4.4)$ & 20 & $9(4.6)$ & $\longleftarrow$ & - & $9.6 \%$ & $-2.40[-5.22,0.42]$ \\
\hline Khoo 2007 & 35 & $5(8.5)$ & 35 & $7(14.75)$ & & & $3.1 \%$ & $-2.00[-7.64,3.64]$ \\
\hline Muller 2009 & 76 & $6.7(4.84)$ & 75 & $10.3(4.97)$ & 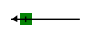 & & $19.2 \%$ & $-3.60[-5.17,-2.03]$ \\
\hline Serclova 2009 & 51 & $7.4(1.3)$ & 52 & $10.4(3.1)$ & $\longrightarrow$ & & $27.1 \%$ & $-3.00[-3.92,-2.08]$ \\
\hline Total $(95 \% \mathrm{CI})$ & 226 & & 226 & & & & $100.0 \%$ & $-2.51[-3.54,-1.47]$ \\
\hline \multicolumn{9}{|c|}{ Heterogeneity: $\mathrm{Tau}^{2}=0.80 ; \mathrm{Chi}^{2}=1 \mathrm{I} .04, \mathrm{df}=5(\mathrm{P}=0.05) ; \mathrm{I}^{2}=55 \%$} \\
\hline \multicolumn{9}{|c|}{ Test for overall effect: $Z=4.76(P<0.0000 I)$} \\
\hline \multicolumn{9}{|c|}{ Test for subgroup differences: Not applicable } \\
\hline & & & & & \multirow[t]{2}{*}{-4} & 2 & 4 & \\
\hline & & & & & & Conver & & \\
\hline
\end{tabular}

\section{ADDITIONAL TABLES}

Table 1. Search strategies

\begin{tabular}{|c|c|c|}
\hline Database & Search strategies & Number of Hits \\
\hline The Cochrane Library & $\begin{array}{l}\text { ((fast AND track) OR (ERAS) OR (En- } \\
\text { hanced AND recovery AND Surgery)) } \\
\text { AND (colorectal OR colon OR Rectum } \\
\text { OR Sigmoid) AND (surgery OR surgical } \\
\text { OR procedure) }\end{array}$ & 22 \\
\hline Pubmed & $\begin{array}{l}\text { ((fast AND track) OR (ERAS) OR (En- } \\
\text { hanced AND recovery AND Surgery) OR } \\
\text { (“fast track")) AND (colorectal OR colon } \\
\text { OR Rectum OR Sigmoid) AND (surgery } \\
\text { OR surgical OR procedure) }\end{array}$ & 125 \\
\hline
\end{tabular}

Fast track surgery versus conventional recovery strategies for colorectal surgery (Review) 
Embase

Search strategy will be conducted through 82 the advanced search feature of EMBASE, with the next options tagged on:

-Map to preferred terminology

-Also search as keyword-Include subterms/derivatives (explosion search)

-1990 - 2009

-EMBASE Only

(((fast and track) or ERAS).mp. or fasttrack.ti,ab. or (enhanced and recovery and surgery).mp.) AND (((resection or surgical or surgically or surgery or laparoscopy or laparoscopic or laparoscopically or laparoscopy).ti,ab. or Surgery/) and (exp colon/ or exp rectum/ or exp sigmoid/ or rectal.ti,ab. or colonic.ti,ab. or colon.ti,ab. or colorectal.ti,ab. or rectum.ti,ab. or sigmoid.ti,ab.) OR exp Colorectal Surgery/ or exp Rectum Surgery/ or exp Colon Surgery/)

AND

exp Randomization/ OR exp Controlled Clinical Trial/ OR Randomized Controlled Trial/ OR random allocation.mp. OR Double Blind Procedure/ OR Single Blind Procedure/ OR Clinical Trial/ OR exp Comparative Study/ OR exp evaluation/ OR exp follow up/ OR exp Prospective Study/ OR control*.ti,ab. OR prospectiv*. ti,ab. OR volunteer*.ti,ab

ISI WEb of Knowledge

( (Fast and Track) OR (ERAS) OR (enhanced AND recovery AND surgery) ) AND (colonic or colorectal OR colon OR rectum OR rectal OR sigmoid) AND (randomized controlled trial OR controlled clinical trial OR randomised controlled trials OR random allocation OR doubleblind method OR single-blind method OR clinical trial OR clinical trials OR clinical trial OR ((singl* OR doubl*OR trebl* OR tripl*) AND (mask* OR blind*)) OR placebos OR placebo* OR random* OR comparative study OR evaluation stud* OR follow-up stud* OR prospective stud* OR control* OR prospectiv* OR volunteer*) 
Table 1. Search strategies (Continued)

NHS Economic Evaluation Database in ((fast AND track) OR (ERAS) OR (En- 3 the Cochrane library hanced AND recovery AND Surgery)) AND (colorectal OR colon OR Rectum OR Sigmoid) AND (surgery OR surgical OR procedure)

Table 2. Patient characteristics

\begin{tabular}{|c|c|c|c|c|c|c|c|c|c|c|c|}
\hline Trial & $\mathbf{N}$ & Age & & $\begin{array}{l}\text { Gender } \\
(\mathrm{M} / \mathrm{F})\end{array}$ & & BMI & & $\begin{array}{l}\text { POS- } \\
\text { SUM }\end{array}$ & & $\begin{array}{l}\text { ASA 1- } \\
2 / 3\end{array}$ & \\
\hline & $\begin{array}{l}\text { ERAS/ } \\
\text { conv }\end{array}$ & ERAS & conv & ERAS & conv & ERAS & conv & ERAS & conv & ERAS & conv \\
\hline $\begin{array}{l}\text { Ander- } \\
\text { son } \\
2003\end{array}$ & $14 / 11$ & $\begin{array}{l}64[55- \\
68]\end{array}$ & $\begin{array}{l}68[65- \\
75]\end{array}$ & $6 / 8$ & $5 / 6$ & $\begin{array}{l}26 \\
28]^{\wedge}\end{array}$ & $\begin{array}{l}24 \\
28]^{\wedge}\end{array}$ & $\begin{array}{l}26[22- \\
27]^{\wedge}\end{array}$ & $\begin{array}{l}26 \\
28]^{\wedge}\end{array}$ & $13 / 1^{\dagger}$ & $10 / 1^{\dagger}$ \\
\hline $\begin{array}{l}\text { Delaney } \\
2003\end{array}$ & $31 / 33$ & $\begin{array}{l}30.6 \\
{[16.9]^{\wedge}}\end{array}$ & $\begin{array}{l}41.9 \\
{[13.3]^{\wedge}}\end{array}$ & $21 / 10$ & $21 / 12$ & * & * & * & * & $3 / 16 / 12$ & $0 / 26 / 7$ \\
\hline $\begin{array}{l}\text { Gatt } \\
2005\end{array}$ & $19 / 20$ & $\begin{array}{l}67 \\
76]\end{array}$ & $\begin{array}{l}67 \quad[60- \\
70]\end{array}$ & $9 / 10$ & $14 / 6$ & $\begin{array}{l}24 \\
29]\end{array}$ & $\begin{array}{l}27[24- \\
30]\end{array}$ & $\begin{array}{l}13[11- \\
15]\end{array}$ & $\begin{array}{l}12[11- \\
16]\end{array}$ & $2[2-2]^{\ddagger}$ & $2[2-3]^{\ddagger}$ \\
\hline $\begin{array}{l}\text { Khoo } \\
2007\end{array}$ & $35 / 35$ & $\begin{array}{l}69 . \\
3[46.3- \\
87.7]\end{array}$ & $\begin{array}{l}73 . \\
0 \quad[46.4- \\
84.6]\end{array}$ & $12 / 23$ & $15 / 20$ & $*$ & * & * & * & $5 / 25 / 5$ & $3 / 27 / 5$ \\
\hline $\begin{array}{l}\text { Muller } \\
2009\end{array}$ & $76 / 75$ & $\begin{array}{l}62 \quad[27- \\
91]\end{array}$ & $\begin{array}{l}59 \\
89]\end{array}$ & $37 / 39$ & $40 / 35$ & $\begin{array}{l}24 \\
35]\end{array}$ & $\begin{array}{l}26 \\
33]\end{array}$ & * & * & $2 / 50 / 24$ & $3 / 54 / 18$ \\
\hline $\begin{array}{l}\text { Serclova } \\
2009\end{array}$ & $51 / 52$ & $\begin{array}{l}35.1[11 . \\
0]^{\wedge}\end{array}$ & $\begin{array}{l}37.6[12 . \\
5]^{\wedge}\end{array}$ & $20 / 31$ & $32 / 20$ & * & * & * & * & * & * \\
\hline
\end{tabular}

conv $=$ conventional treatment protocol

$\hat{\imath}=$ mean $[\mathrm{SD}]$

$*=$ unreported

$\dagger=$ ASA 1 en 2 taken together.

$\ddagger=$ presented as median [interquartile range] 


\section{H I S T O R Y}

Protocol first published: Issue 1, 2009

Review first published: Issue 2, 2011

\section{CONTRIBUTIONSOFAUTHORS}

- W.R Spanjersberg. Writing manuscript, methodological setup, developing search strategies, performing literature search, evaluate identified trials, statistical analysis.

- J.C Reurings. Second reviewer, abstract selection, manuscript revision.

- F. Keus. methodological and statistical support, manuscript revision

- CJ.H.M v Laarhoven. Supervision of manuscript preparation, revision of manuscript, mediation in differences by reviewers.

\section{DECLARATIONSOF INTEREST}

none

\section{DIFFERENCES BETWEEN PROTOCOLANDREVIEW}

In the protocol we described that when selecting non randomised trials, methodological quality would be assessed by use of MINORS. However, since 6 RCT's were identified, no non- or pseudo- randomised trials were included in this review.

\section{NDEX TERMS Medical Subject Headings (MeSH)}

Colonic Diseases [* ${ }^{*}$ surgery]; Early Ambulation; Length of Stay; Pain, Postoperative [drug therapy]; Patient Readmission [statistics \& numerical data]; Postoperative Care [adverse effects; * methods; mortality]; Randomized Controlled Trials as Topic; Recovery of Function; Rectal Diseases [* surgery]

\section{MeSH check words}

Humans 